\title{
High-Fidelity Buckling Analysis of Composite Cylinders using the STAGS Finite Element Code
}

\author{
Mark W. Hilburger ${ }^{*}$ \\ NASA Langley Research Center, Hampton, Virginia, 23681, USA
}

\begin{abstract}
Results from previous shell buckling studies are presented that illustrate some of the unique and powerful capabilities in the STAGS finite element analysis code that have made it an indispensable tool in structures research at NASA over the past few decades. In particular, prototypical results from the development and validation of high-fidelity buckling simulations are presented for several unstiffened thin-walled compression-loaded graphite-epoxy cylindrical shells along with a discussion on the specific methods and user-defined subroutines in STAGS that are used to carry out the high-fidelity simulations. These simulations accurately account for the effects of geometric shell-wall imperfections, shell-wall thickness variations, local shell-wall ply-gaps associated with the fabrication process, shell-end geometric imperfections, nonuniform applied end loads, and elastic boundary conditions. The analysis procedure uses a combination of nonlinear quasi-static and transient dynamic solution algorithms to predict the prebuckling and unstable collapse response characteristics of the cylinders. Finally, the use of high-fidelity models in the development of analysis-based shell-buckling knockdown (design) factors is demonstrated.
\end{abstract}

\section{Introduction}

$\mathrm{T}$ he ever-increasing need to produce lighter-weight aerospace shell structures has led to the use of highly optimized structural designs and advanced material systems. Buckling is often a primary failure mode that drives the design of light-weight aerospace shell structures such as cryogenic tanks and interstage structures in launch vehicles. In particular, maximum structural efficiency typically necessitates the use of thin highly stressed skins that can be susceptible to buckling failures when subjected to compression loads. Thus, buckling is an important and often critical consideration in the design of these structures and reliable, validated high-fidelity analysis tools and design criteria are needed.

The general topic of shell buckling has garnered a tremendous amount of attention since the beginning of the $20^{\text {th }}$ century. From approximately 1930 to 1967 , many shell-buckling experiments were conducted on isotropic unstiffened cylindrical shells under a variety of loading conditions. Typically, the experiments yielded buckling loads that were substantially lower than the corresponding analytical predictions, which were based on simplified linear bifurcation analyses of geometrically perfect shells with nominal dimensions and idealized boundary conditions. The pioneering works by von Kármán and Tsien $(1941)^{1}$, by Donnell and Wan $(1950)^{2}$, and by Koiter $(1945)^{3}$ identified small deviations from the idealized geometry of a

* Senior Research Engineer, Structural Mechanics and Concepts Branch, Hampton, VA, 23681, USA, Senior Member AIAA. 
shell, known as initial geometric imperfections, as a primary source of discrepancy between corresponding analytical predictions and experimental results. However, the analysis capabilities at that time could not meet the intense computational requirements of the nonlinear analyses needed to predict accurately the imperfection sensitivity and buckling loads of the tested shells. Thus, designers often used a simplified analysis procedure with empirical data to develop safe designs for buckling-critical structures. Specifically, the traditional approach for designing thinwalled buckling-resistant isotropic shell structures is to predict the buckling load of the shell with a linear bifurcation buckling analysis, and then to reduce this predicted load with an empirical "knockdown" factor. The empirical knockdown factor is intended to account for the difference between the predicted buckling load and the actual buckling load for the shell determined from tests and is often based on the "lower bound" design recommendations reported in the NASA SP-8007 document (1968). ${ }^{4}$ This approach to shell buckling design remains prominent in industry practice, as evidenced by the extensive use of the NASA space vehicle design criteria. ${ }^{4-8}$

From approximately 1960 to 1989 , digital computers emerged and matured into a practical tool for research and design. With these computational tools, considerable effort was put forth to determine analytically the effects of initial geometric imperfections on the buckling response of shells. These efforts were largely based on Koiter's asymptotic theory ${ }^{3,9}$ for shells with nominal dimensions and idealized boundary conditions. In addition, in-depth experimental investigations were conducted at institutions such as the NASA Langley Research Center and the Wright Aeronautical Laboratories at Wright-Patterson Air Force Base, in addition to various academic institutions worldwide. It was during this time period that the measurement of initial geometric imperfections in test specimens and high-fidelity response measurement techniques and apparatus were recognized as important elements in the development of design criteria for buckling of shells. ${ }^{10-13}$

Another significant development that occurred in the 1960s to 1980s was the development of computational structural analysis codes based on finite-difference or finite-element methods. Two prominent examples at the forefront in the development of this technology are the NASA Structural Analysis code $\left(\mathrm{NASTRAN}^{14}\right.$ ) and the Structural Analysis of General Shells code $\left(\right.$ STAGS $\left.^{15}\right)$. These codes evolved over many years and now possess robust static and dynamic nonlinear structural analysis capabilities, along with extensive graphics capabilities, that enable scientists and engineers to model and visualize experiments or actual flight hardware to an enormous level of detail. Prototypical examples of the application of the STAGS code can be seen in the in-depth, highly detailed studies of cylinder buckling response and imperfection sensitivity documented in Refs. 16-18 and the nonlinear and buckling analyses of the Space Shuttle super-light-weight external fuel tank that is documented in Refs. 19-23.

The results from these studies showed that highly accurate predictions of the buckling response of thin-walled cylinders can now be achieved based on detailed nonlinear analysis and that analysis-based knockdown factors could become a viable replacement for the test-based design knockdown factors used currently. In addition, the robust nonlinear solution algorithms and user-defined subroutines enabled precise, systematic and efficient exploration of the imperfection sensitivities that were affecting the structural response.

Based on these promising results, Hilburger and Starnes proposed an analysis-based design 
approach in 2003 that can accurately account for the effects of initial geometric imperfections. ${ }^{24}$ With this approach, measured initial geometric imperfection data from a representative set of cylindrical shells are used to determine a manufacturing-process-specific imperfection signature for these shells. This imperfection signature is then used as input into nonlinear finite-element analyses. The imperfection signature represents a "first-approximation" imperfection shape that is suitable for developing preliminary-design data. The STAGS code, again, played a prominent role in developing the analysis-based design strategy.

In recent times, this new analysis-based approach has been further developed and used to generate improved design knockdown factors for NASA's next generation of heavy-lift launch vehicle shell structures. ${ }^{25}$ These new factors have been developed for a particular class of integrally stiffened metallic cylinders and account for manufacturing-process-specific imperfection signature as well as critical design features such as longitudinal welds which have been shown to significantly affect the buckling response of these shells. The STAGS finite element code was used extensively in the path-finding work during the development of these new knockdown factors as well as providing benchmark results to help develop and validate models using other commonly used commercial finite element codes.

The present paper is intended to highlight some of the ground-breaking analysis capabilities of the STAGS finite-element code that have been developed over the past few decades and that are enabling new design technologies such as analysis-based shell buckling knockdown factors. To this end, selected results from previous numerical and experimental studies on the buckling of unstiffened thin-walled compression-loaded graphite-epoxy cylindrical shells are presented. First, a set of three composite laminated cylindrical shell test articles are described. Then, a high-fidelity nonlinear shell-analysis procedure is describe and includes details on how various modeling details are included in the STAGS model including initial geometric shell-wall imperfections, shell-wall thickness variations, shell-end geometric imperfections, nonuniform applied end loads, and elastic boundary conditions. Next, selected test and analysis correlation data is presented to illustrate the accuracy of the high-fidelity models. Finally, the foundational work towards the development and implementation of new analysis-based shell buckling knockdown factors are described and design implications are discussed.

\section{Test Specimens, Imperfection Measurements and Test Procedure}

\section{Test Specimens}

The test specimens discussed in this paper were fabricated from 0.005-in.-thick AS4/3502 graphite-epoxy pre-impregnated unidirectional tape material made by Hercules, Inc. The nominal unidirectional lamina properties of a typical 0.005-in.-thick ply with a fiber volume fraction of 0.62 are as follows: longitudinal modulus $\mathrm{E}_{1}=18.5 \mathrm{Msi}$, transverse modulus $\mathrm{E}_{2}=1.45 \mathrm{Msi}$, inplane shear modulus $\mathrm{G}_{12}=0.813 \mathrm{Msi}$, and major Poisson's ratio $v_{12}=0.30$. The material was laid up on a 15.75-in.-diameter mandrel and cured in an autoclave to form three shells with different laminates including an 8-ply axially stiff $\left[-45 /+45 / 0_{2}\right]_{\mathrm{s}}$ laminate, an 8-ply circumferentially stiff $\left[-45 /+45 / 90_{2}\right]_{\mathrm{S}}$ laminate, and an 8-ply quasi-isotropic $[-45 /+45 / 0 / 90]_{\mathrm{S}}$ laminate. The resulting three shells are referred to herein as shells or specimens $\mathrm{C} 1, \mathrm{C} 2$, and $\mathrm{C} 3$, respectively. These specimens had a nominal length of $16.0 \mathrm{in}$. and a nominal radius of $8.0 \mathrm{in}$. 
The specimens had a nominal shell-wall thickness of $0.04 \mathrm{in}$. and a shell-radius-to-thickness ratio of 200. Both ends of the specimens were potted in an aluminum-filled epoxy resin to assure that the ends of the specimens did not fail prematurely during the test. The potting material extended approximately 1.0 inch along the length of the specimens at each end resulting in a test section that was approximately $14.0 \mathrm{in}$. long. The ends of the specimens were machined flat and parallel to assure proper load introduction during the tests. A photograph of a typical specimen is shown in Figure 1 and the specimen coordinate system and geometry is shown in Figure 2. The shell length, test-section length, radius, and thickness are designated as $L, L_{T}, R$ and $t$, respectively.

\section{Imperfection Measurements}

Three-dimensional surveys of the inner and outer shell-wall surfaces of the specimens were made prior to testing to determine the initial geometric imperfection shapes of the shell-wall and shell-wall thickness distributions. Measurements were taken over a uniform grid with increments of $0.125 \mathrm{in}$. in the axial direction and approximately $1^{\circ}$ of arc in the circumferential direction over the exposed surfaces of the specimens. The inner surface measurement was used to determine the initial geometric imperfection shape of a specimen, and the difference between the outer and inner surface measurements was used to determine the shell-wall thickness distribution. A contour plot of the normalized initial geometric imperfection for specimen C3 is shown in Fig. 3. The measured geometric imperfection $w_{o}$ is normalized by the average measured shell-wall thickness $t_{\text {ave }}=0.0381$ inches. These results indicate that the initial geometric imperfection is periodic in the circumferential direction and has slight deviations along the axial direction. The amplitude of the imperfection varies from $+1.341 t_{\text {ave }}$ to $-1.535 t_{\text {ave }}$. A contour plot of the normalized shell-wall thickness variation for specimen C3 is shown in Fig. 4 , where the measured thickness value $t_{o}$ is normalized by the average measured shell-wall thickness $t_{\text {ave }}$. These results indicate that the shell-wall thickness, and hence the laminate stiffnesses, varies significantly over a short distance. The thickness varies from 0.928 to 1.321 times $t_{\text {ave }}$. Most of the thickness variation is attributed to local variations in the resin content of the laminate associated with the fabrication process. However, the dark blue angular pattern in the thickness distribution is attributed to small gaps between adjacent pieces of graphite-epoxy tape in some of the laminate plies that were generated during the lay-up and curing processes. Such a region is referred to herein as a lamina ply-gap or a ply-gap. In the particular case of shell C3, such a locally thin region in the shell wall consists of a 7-ply-thick laminate rather than the nominal 8-ply-thick laminate. For the case where one ply-gap intersects another ply-gap, the shell wall consists of a 6-ply-thick laminate. Moreover, these locally thin shell-wall regions have a significant shell-wall mid-surface eccentricity. A circumferential ply-gap is caused by a gap between two adjacent $90^{\circ}$ pieces of tape, a helical ply-gap is caused by a gap between two adjacent $45^{\circ}$ pieces of tape, and an axial ply-gap is caused by a gap between two adjacent $0^{\circ}$ pieces of tape. Magnified cross-sectional views of typical ply-gaps in a $-45^{\circ}$ outer ply of a specimen laminate and in a $90^{\circ}$ mid-surface ply are shown in Figs. 5a and 5b, respectively. The widths of the ply-gaps shown in Figs. $5 \mathrm{a}$ and $5 \mathrm{~b}$ are on the order of 0.02 in. or approximately equal to half the shell-wall thickness, and the ply-gap depth is approximately 0.005 in. or approximately equal to the nominal lamina ply thickness. Lamina ply-gaps with gap widths as large as 0.1 in. have been observed in some of the shell specimens. The red colored longitudinally aligned patterns in the thickness contour plot are caused by locally thickened regions of the outermost plies of the laminate that develop during the curing process to form 
outer shell-wall surface ridges. A magnified cross-sectional view of such a region is shown in Fig. 5c. Some of the shell-wall thickness features, such as lamina ply-gaps, are smaller than the imperfection-measurement grid spacing used in this study, and as a result, some of the smaller thickness variation features may not have been included in the measurements.

Measurements of the top and bottom loading surfaces of the specimens were made every degree around the circumference of the specimens to determine the variation in the shell-end or loadingsurface geometry. Typical top and bottom shell-end or loading-surface geometry variations for specimen $\mathrm{C} 3$, denoted by $\delta_{t o p}(\theta)$ and $\delta_{b o t}(\theta)$, respectively, are shown in Fig. 6. The maximum amplitude of this loading-surface variation is approximately 0.0015 inches, which is approximately $0.01 \%$ of the specimen length.

\section{Test Apparatus and Procedure}

The specimens were instrumented with electrical resistance strain gages, and direct-current differential transducers (DCDT's) were used to measure displacements. Three non-collinear DCDT's were positioned at three corners of the upper loading platen of the test machine and used to measure the end-shortening displacement $\Delta$ and the rotations $\phi_{y}$ and $\phi_{z}$ of the loading platen as illustrated in Fig. 2. Typical measured platen rotations are shown in Figure 7. To control the load introduction into the specimens, the upper loading platen was aligned with the loading surface of the specimen as well as possible before the test by adjusting leveling bolts in the corners of the upper platen until strains measured by selected strain gages on the specimens indicated a uniform axial strain distribution around the circumference of the shell. The specimens were loaded in compression with a 300,000-lb hydraulic universal-testing machine by applying an end-shortening displacement to the loading surfaces of the specimens. The specimens were loaded until general instability or failure of the shells occurred.

A shadow moiré interferometry technique was used to observe the shell-wall prebuckling, buckling and post-buckling radial deformation patterns. All data were recorded with a data acquisition system, and the moiré patterns were recorded photographically and on videotape.

\section{Finite-Element Models and Analysis Procedure}

The shells considered in this study were analyzed with the STAGS (STructural Analysis of General Shells) nonlinear shell analysis code. ${ }^{15}$ STAGS is a research-oriented finite-element code developed for the nonlinear static and dynamic analysis of general shells, and can include the effects of geometric and material nonlinearities in the analysis. It possesses a typical suite of elements including beams, plates, and solids, elastic and rigid links, as well as generalized contact elements, sandwich elements, and user-defined element routine similar to that in Abaqus. In addition, STAGS includes progressive damage analysis capabilities including crack propagation, delamination modeling via decohesion elements and property degradation methods for composite materials. The STAGS code also has a unique feature in that it includes several different user-defined subroutines that can be linked to the STAGS program executable to accomplish many different modeling and data processing tasks. In addition, the STAGS source code is open for modification by the user, which enables the implementation of new solution 
techniques directly into the code. The user-written subroutine capability is used extensively by NASA researchers and in the work presented herein.

\section{Finite-Element Models}

A typical finite-element model of a specimen is illustrated in Fig. 2. The standard 410 quadrilateral shell element from the STAGS element library was used in the models. The element is based on the Kirchoff-Love shell theory and the nonlinear Lagrangian strain tensor. The elements of the finite-element mesh are approximately 0.2-in. by 0.2-in. square. Each element has four integration points, which are distributed in such a way as to provide a modeling resolution of approximately 0.1 -in. by 0.1 -in square. This integration point spacing is on the order of the measurement-point spacing used to measure the initial geometric imperfections of the specimens. This highly refined mesh is necessary to model rapidly varying geometric and material parameters such as nonuniform shell-wall thicknesses and lamina stiffness properties. A typical finite-element model contained approximately 100,000 degrees of freedom.

Results from geometrically perfect and imperfect shells are presented in this paper. The geometrically perfect shells are modeled assuming nominal shell geometry, laminate thickness, lamina mechanical properties, and boundary conditions. The boundary conditions consist of setting the circumferential and radial displacements $v$ and $w$ equal to zero in the 1.0-in.-long potted boundary regions of the shell illustrated in Fig. 1, setting $u(L / 2, \theta)=0$, and applying a uniform end-shortening $u(-L / 2, \theta)=\Delta$.

The geometrically perfect finite-element models were then modified to include the effects of the measured shell imperfections in order to simulate the response of the specimens more closely. These modeling modifications included the effects of the measured initial geometric shell-wall imperfections, shell-wall thickness variations, local shell-wall lamina ply-gaps, thicknessadjusted lamina properties, elastic boundary conditions, shell-end geometric imperfections, and nonuniform end loads.

The initial geometric shell-wall imperfection $w_{o}(x, \theta)$ is included in the finite-element models by introducing an initial radial perturbation to each node of the mesh by using the user-written subroutine DIMP available in STAGS. The DIMP subroutine statement is as follows (variables used in the subroutine are highlighted in bold font):

subroutine DIMP(iunit, inode, prop, $X Y Z g, X Y s, v v)$

where inode is the node number from the model, $X Y S$ is a vector that contains the cylindrical coordinate values $x$ and $\theta$ for the node, and $v v$ is the node perturbation vector $\{v v(1), v v(2), \ldots$, $v v(6)\}$ that is used to update the location of the node. Variables $v v(1), v v(2)$ and $v v(3)$ correspond to perturbations to the axial, circumferential, and radial coordinates, respectively, and $v v(4)$, $v v(5)$ and $v v(6)$ correspond to perturbations in the rotations about the axial, circumferential, and radial coordinate directions. During execution, the DIMP subroutine first reads in the measured imperfection data $w_{o}(x, \theta)$ (e.g., data in Figure 3) from an external file and stores it in an array or look-up table. Then DIMP obtains finite-element nodal coordinate values $x$ and $\theta$ for a given node from the $X Y s$ array. A linear interpolation algorithm is used in DIMP to calculate the value 
of the radial perturbation $v v(3)$ for each finite-element node based on the nodal coordinate values and the measured imperfection data stored in the look-up table. Similar calculations are also performed in DIMP to apply local rotations of the finite-element node that are associated with local changes in shell-wall curvature due to the imperfection, $v v(4), v v(5)$. This process is repeated for each node called by the subroutine. The iunit, inode, prop, $X Y Z g$ variables were not used. The actual code inside the subroutine is not reported here for brevity but can be found in Appendix D of Reference 26.

Similarly, the shell-wall thickness $t$, mid-surface eccentricity $e c z$, and lamina material properties $E_{1}, G_{12}, v_{12}$ and $E_{2}$ are adjusted at each integration point of each element in the finite-element models by using the user-written subroutine WALL. The WALL subroutine uses the following subroutine statement and common block for passing modeling information between the subroutine and the main STAGS program (variables used in the subroutine are highlighted in bold font):

subroutine WALL(iunit, ielt, kelt, XYZg, XYs, zeta, ecz, ilin, iplas)

COMMON/WALL1/tL, e1L, u12L, $g L, e 2 L$

During execution, the WALL subroutine first reads in the measured thickness data $t_{o}(x, \theta)$ (e.g., data in Figure 4) from an external file and stores it in an array or look-up table. Then the WALL subroutine obtains finite-element integration point coordinate values $x$ and $\theta$ from the $X Y s$ array. A linear interpolation algorithm is used in WALL to calculate the individual ply thicknesses at each finite-element integration point based on the integration point coordinate values and the measured thickness data stored in the look-up table and are then adjusted using the $t L$ vector (the size of the vector is based on the total number of plies, in this case, eight) The thickness of each lamina ply is assumed to be equal to the total measured laminate thickness divided by the total number of plies in the laminate, $t L(i)=t_{o}(x, \theta) / 8$ where $i=1,8$. The shell-wall mid-surface eccentricity $e c z$ is calculated relative to the average measured shell-wall mid-surface as illustrated in Fig. 8; that is, $\operatorname{ecz}(x, \theta)=-0.5\left(t_{\text {ave }}-t_{o}(x, \theta)\right)$. The updated lamina material properties $\mathrm{E}_{1}, \mathrm{G}_{12}, v_{12}$ and $\mathrm{E}_{2}$ are provided via the $e 1 L(i), u 12 L(i), g L(i)$, and $e 2 L(i)$ vectors, respectively, and calculated based on the rule of mixtures. In general, the rule-of-mixtures calculations used here assumed that any variation in the lamina ply thickness from the nominal thickness is due to a variation in resin volume only, and that the fiber volume remains constant for each ply.

Several simplifying assumptions and approximations were made in modeling the geometry and stiffnesses of a lamina ply-gap detail. First, the finite-element models are limited to modeling the shell-wall thickness variation as discrete changes, as illustrated in Fig. 8, and the resolution of the thickness variation is limited by the finite-element integration point spacing (e.g., 0.1 in.). Results from a study illustrating the potential effects of these modeling approximations and mesh refinement on the response of a shell with a ply-gap indicated that this modeling approach can affect the predicted response. ${ }^{17}$ As one might expect, these modeling approximations can lead to an artificial increase in the width of a lamina ply-gap, resulting in misrepresentation of the local bending stiffnesses and the mid-surface eccentricity of the shell wall at that particular point. In addition, the mesh refinement and integration point spacing used in the present models tend to provide models that are overly stiff in bending by a small amount when they are used for 
modeling a ply-gap detail. However, these modeling approximations were shown to have a relatively small influence on the local bending response of the shell and only resulted in a $2 \%$ variation in the predicted buckling loads for the shells considered herein.

The second assumption is related to the modeling of the stiffnesses of the lamina ply-gap detail. Two modeling approaches were considered. One approach is to model each ply-gap with a local reduction in thickness, as measured, including the local eccentricity $e c z$, and reducing the appropriate number of lamina plies in the shell-wall laminate model, hence, reducing the local stiffnesses associated with the local ply-gap detail. The second approach is to model the ply-gap with the local as-measured reduced shell-wall thickness and the corresponding local mid-surface eccentricity, but assume that the local thickness reduction is due to a reduction in the resin volume only and, consequently, keeping the fiber volume constant. This approach neglects the local stiffness reduction associated with the reduced number of plies. However, results from a study of this modeling assumption indicated that neglecting the local stiffness reduction associated with a ply-gap would cause only slight differences in the magnitude of the local bending response and no more than a $2 \%$ variation in the predicted buckling load for a compression-loaded shell. ${ }^{17}$ In addition, the results indicated that the local shell-wall mid-surface eccentricity is the most important feature of the ply-gap detail for these stability critical problems. As a result, the latter modeling approach was used in the present study.

To provide a better simulation of the constraints provided by the potting material at the ends of the specimens, effective axial and radial potting-support stiffnesses were determined for each shell specimen using a two-dimensional generalized plane-strain finite-element analysis of the potting-material-shell-wall detail shown in Fig. 9a. Material properties of the potting compound were characterized by Iosipescu tests of the potting material reported by Weiland et al. ${ }^{27}$ The nominal properties of the potting material are as follows: Young's modulus $\mathrm{E}=1.15 \mathrm{Msi}$, shear modulus $\mathrm{G}_{12}=0.36 \mathrm{Msi}$, and Poisson's ratio $v=0.59$. The effective laminate axial stiffness $\mathrm{E}_{\mathrm{x}}$ was used in the shell-wall models which include $\mathrm{E}_{\mathrm{x}}$ equal to 11.83, 3.60 and $8.07 \mathrm{Msi}$, for shells $\mathrm{C} 1, \mathrm{C} 2$ and $\mathrm{C} 3$, respectively. To determine the effective axial and radial potting-support stiffnesses, two numerical experiments were conducted using this local model. In the first numerical experiment, an axial displacement $\Delta$ was applied to the unpotted end of the shell model, and the resulting predicted axial strain response, shown in Fig. 9b, was used to calculate an effective axial stiffness $K_{A}$ for the portions of the shell wall supported by the potting material. The predicted effective axial potted-shell stiffnesses are 26.55, 6.73 and 26.26 Msi for shells C1, $\mathrm{C} 2$ and $\mathrm{C} 3$, respectively. In the second numerical experiment, a unit force $F_{z}$ was applied to the shell model, and the resulting $w$ (radial) displacement and nodal force at the junction of the shell wall and the potting material was used to calculate an effective radial support stiffness $K_{R}$. The effective radial potting-support stiffness was predicted to be approximately equal to $1.0 \mathrm{E} 5 \mathrm{lbf} / \mathrm{in}$. The radial support stiffness was incorporated into the models via linear spring elements in STAGS.

Nonuniform end loading of a specimen is attributed to initial specimen-end or loading-surface imperfections (see Figure 6) and to upper loading-platen rotations that are measured during the experiment. A typical set of measured loading platen rotations is shown in Fig. 7 and indicate that significant upper platen rotations occur from the onset of loading up to a load value of approximately 6,000 lbs. These rotations are attributed to an initial settling of the upper loading 
platen onto the specimen. The rotations of the movable upper loading platen reach a steady state at a load value of 6,000 lbs., and the loading of the specimen, for the most part, continues without additional upper loading platen rotations from 6,000 lbs. up to the buckling load. During the buckling event, the upper loading platen undergoes an additional amount of rotation.

These nonuniform displacements were simulated in the finite-element model in three parts. First, the measured upper and lower loading-surface imperfections $\delta_{\text {top }}(\theta)$ and $\delta_{\text {bot }}(\theta)$, respectively, were included in the finite-element model by introducing an initial in-plane axial perturbation $v v(1)$ to each of the nodes at the loaded ends of the shell using the DIMP subroutine. Then, the compression load was applied to the shell in two parts. The nonuniform loadingsurface imperfections, $-\delta_{t o p}(\theta)$ and $-\delta_{b o t}(\theta)$, were applied as displacements to the upper and lower ends of the shell, respectively, in the first load step of the analysis to simulate a full contact condition between the shell ends and the loading platens. Then, the experimentally measured end-shortening displacement $\Delta$ and upper loading-platen rotations $\phi_{y}$ and $\phi_{z}$ were applied to the upper loading surface while holding the lower loading surface fixed as illustrated in Fig. 2; that is, $u(-L / 2, \theta)=\Delta+R \cos \phi_{y} \cos \theta+R \cos \phi_{z} \sin \theta-\delta_{\text {top }}(\theta)$ and $u(L / 2, \theta)=-\delta_{\text {bot }}(\theta)$.

These nonuniform loads were applied by using a user-defined subroutine USRLD in STAGS. The USRLD subroutine uses the following subroutine statement and call statement to define the applied displacement (variables used in the subroutine are highlighted in bold font):

subroutine USRLD(iunit, $\boldsymbol{X}, \boldsymbol{Y}$, nrows, ncols, isys)

call FORCE $(\boldsymbol{P}, \boldsymbol{l t}, \boldsymbol{l d}, \mathrm{li}, \mathrm{lj}, \mathrm{lax})$

where $X$ and $Y$ correspond to the $x$ and $\theta$ coordinates of the finite-element node and isys corresponds to the load set number. At the time of this work, the user was able to define two separate load sets in STAGS, isys $=1$ was an active load set and $i s y s=2$ was a passive load set. The subroutine FORCE is called inside USRLD to define the load for a particular node and includes the load magnitude $P$, load type $l t$, and load direction $l d$. The load type can include applied displacement, applied point force, distributed load, live pressure, and thermal.

Finally, the effects of selected specimen parametric uncertainties were determined based upon the results of a traditional combinatorial analysis of the effects of selected specimen parametric uncertainties. ${ }^{17}$ The parametric uncertainties considered include uncertainties in the imperfection measurements, the lamina fiber volume fraction, and the applied load distribution. The imperfection measurement uncertainties are attributed to the accuracy tolerances of the coordinate measurement device used to measure the initial geometry of the shell. The shell-wall imperfection measurement values and the shell-end-shape imperfection measurement values used in the present study are accurate to within 0.0006 inches, or $0.75 \mathrm{E}-4 R$ and $1.5 \mathrm{E}-4 L$, where $R$ and $L$ are the shell radius and length, respectively. The shell-wall thickness values are accurate to within 0.0012 inches, or $0.03 t_{\text {nom }}$ ( $t_{\text {nom }}$ is the nominal laminate thickness equal to 0.04 inch). The lamina fiber volume fraction is specified by the manufacturer of the material to be equal to 0.65 \pm 0.03 for the 0.005 -in.-thick graphite-epoxy preimpregnated tape material used to fabricate the test specimens. Applied load distribution uncertainties are measured indirectly by monitoring the measured and predicted axial strains at selected points near the top and bottom loading surfaces of the shell. A correction to the applied displacements can be determined from the differences in 
the measured and predicted strains as follows. A user-written program external to the STAGS code was used to analyze the differences in the measured and predicted strains for a specified applied load value. This program used an iterative predictor-corrector method to determine a correction to the applied shell-end displacements. A new finite-element analysis was conducted with this displacement correction included in the model. This process was repeated iteratively until the difference in the measured and predicted strains reached a predetermined tolerance. A typical load correction is shown in Figure 10.

\section{Nonlinear Analysis Procedure}

The code uses both the modified and full Newton methods for its nonlinear solution algorithms, and accounts for large rotations in the shell by using a corotational algorithm at the element level. The Riks pseudo arc-length path-following method ${ }^{28}$ is used to continue a solution past the limit points of a nonlinear response. With this strategy, the incrementally applied loading parameter is replaced by an arc-length along the solution path, which is then used as the independent loading parameter. The arc-length increments are automatically adjusted by the program as a function of the solution behavior. The transient analysis option in STAGS uses proportional structural damping and an implicit numerical time-integration method developed by Park. ${ }^{29}$ Additional information on the transient analysis procedure can be found in Reference 30 by Riks, Rankin and Brogan. It should be noted that these advanced nonlinear solution algorithms (e.g., the corotational algorithm, Riks arc-length method, transient dynamic analysis, etc.) were first developed for STAGS and represented significant breakthroughs in the analysis for thin-walled shells at the time of their development, many of which have since been migrated to commercial finite-element codes.

The prebuckling, buckling and postbuckling responses of the shells presented herein were determined using the following analysis procedure. A modified version of Riks pseudo arclength path-following method in STAGS was used to compute the initial shell response until just before buckling occurred. The Riks subroutine was modified inside the STAGS sourcecode so that it could monitor the number of negative roots in the tangent stiffness matrix and make adjustments to the solution. More specifically, when a negative root was identified, indicating an unstable equilibrium solution, the solution was discarded and the analysis was automatically restarted from the previous stable equilibrium solution and would proceed with a reduced step size. This process would continue until a solution close the limit point was obtained based on a user-specified solution tolerance (typically less than $0.5 \%$ of the expected critical endshortening). The unstable buckling response of the shell was predicted using the nonlinear transient analysis option of the STAGS code. The transient analysis was initiated from an equilibrium state close to the limit point by incrementing the end displacement by a small amount. An initial time step of 1.0E-8 seconds was used in the analysis, and the time step was automatically adjusted by the program as a function of the solution behavior. The transient analysis was continued until the kinetic energy in the shell had dissipated to a negligible level,

which indicated that the transient response had attenuated. Once the transient analysis had attenuated to a near-steady-state solution, a quasi-static load relaxation option of the code was used to establish a stable postbuckling equilibrium state. 


\section{Results and Discussion}

Numerically predicted and experimentally measured results for three compression-loaded 8-ply graphite-epoxy laminated cylindrical shells are presented in this section. The shell-wall laminates include two different orthotropic laminates and one quasi-isotropic laminate. The predicted results were obtained from finite-element models of geometrically perfect shells and shell models that include initial geometric shell-wall imperfections, shell-wall thickness variations and thickness-adjusted lamina properties, local shell-wall lamina ply-gaps, elastic boundary support conditions, and nonuniform loading effects, as described in section III, referred to herein as the imperfect shell. In addition, some models also include the effects of specimen parametric uncertainties. The results presented include predicted and measured load-end-shortening response curves, predicted and measured load-strain response curves, and predicted prebuckling, buckling and postbuckling deformation response patterns.

\section{Predicted and Measured Response Comparisons}

Analytically predicted and experimentally measured load-end-shortening response curves for shells C1, C2 and C3 are presented in Fig. 11. Values of the axial load $P$ and the end-shortening $\Delta$ are normalized by the linear bifurcation buckling load of quasi-isotropic shell C3, $P_{b i f}^{q}=42,590$ lbs., and the nominal shell-wall thickness $t_{\text {nom }}=0.04$ in., respectively. The solid and dashed lines in the figure represent experimentally measured and analytically predicted results, respectively. Each shell has two predicted response curves representing analytically predicted upper and lower bounds to the imperfect shell response based on specimen parametric uncertainties described in section III. The measured results indicate that shells C1, C2 and C3 exhibit global buckling at normalized load values of 0.652, 0.749 and 0.803, respectively, and are 7.8, 13.7 and 17.6\% lower than the predicted loads for the corresponding geometrically perfect shells, respectively. The global buckling response of the shell is characterized by a sudden reduction in the axial load in the shell that is associated with the transient collapse. These results indicate that, for the most part, the measured load-end-shortening response curves for the shells fall on or within the predicted response bounds.

The load-time history of the predicted transient collapse response of the imperfect shell C3 is shown in Fig. 12. For this shell model, buckling occurs at a normalized load of 0.890. The loadtime history curve exhibits a sudden reduction in axial load until the collapse response attenuates and the axial load achieves a steady-state value. The kinetic energy in the shell obtains a maximum value during the transient collapse response and dissipates over time and the shell reaches a stable postbuckling equilibrium state after approximately 0.007-0.008 seconds. The shell exhibits postbuckling load-carrying capacity, however, the effective axial stiffness of the specimen is significantly reduced in the postbuckling range of loading. In addition, the results indicate that the postbuckling load level is relatively insensitive to the different types of imperfections considered herein.

The corresponding transient deformation responses for selected time steps during the transient collapse response of the imperfect shell $\mathrm{C} 3$, indicated by the letters A through D in Fig. 12, are presented in Fig. 13a through 13d, respectively. Just before buckling occurs, the shell-wall deformations are characterized by several localized ellipse-like buckles located near one end of 
the shell, as indicated in Fig. 13a. The localization in the deformation pattern is caused by the combination of a local geometric shell-wall imperfection and the bending boundary-layer deformations that form near the end of the shell. After approximately 0.0012 seconds have elapsed in the transient response, a single ellipse-like buckle has grown in amplitude and interacts with the destabilizing stresses in the shell wall to cause the general instability and collapse of the shell. The magnitude of the shell-wall radial displacement varies between $\pm 0.5 t_{\text {nom }}$ at this point in the response. After additional time has elapsed in the transient collapse response, additional local buckles have formed around the circumference and along the length of the shell, as indicated in Fig. 13c, and the normalized axial load has decreased from 0.890 to 0.554 . In addition, some of the buckles in the shell begin to coalesce into larger diamond-shaped buckles. The magnitude of the shell-wall radial displacement varies between $+3 t_{\text {nom }}$ to $-7 t_{\text {nom }}$. After approximately 0.01 seconds have elapsed in the transient response, the kinetic energy in the shell has dissipated to a negligible level indicating that the transient response has attenuated, and the shell has deformed into a stable postbuckling mode-shape, as indicated in Fig. 13d.

Predicted initial post-collapse normal displacement contours and the corresponding observed moiré fringe patterns for shell C2 (qualitatively similar to the observed response for C3) are shown in Fig. 14. These results indicate that the shell collapses into a diamond-shaped global buckling pattern with 14 half-waves around the circumference and two half-waves along the length, as predicted by the transient analysis. In addition, the predicted mode-shape is in-phase with the observed mode-shape. Similar results for shell C3 indicate that the shell collapses into a diamond-shaped pattern with 16 circumferential half-waves and two axial half-waves. However, the analytical results predicted that the deformation pattern can change its circumferential alignment by approximately $15^{\circ}$ depending on the values of the parametric uncertainties used in the analysis.

Analytically predicted and experimentally measured back-to-back strain response curves for a representative location on shell C3 are presented in Fig. 15. Values of the axial strain $\varepsilon_{x}$ are normalized by the axial strain at buckling $\varepsilon_{c r}=0.00257$. The strain results represent back-toback surface strain measurements within the bending boundary layer near one end of the shell from strain gages 83 and 84 . These gages are outer and inner surface strain gages located at $x / L_{T}$ $=0.071$ and $\theta=180^{\circ}$. The bending boundary layer behavior is evident in the divergent character of the back-to-back predicted and measured strain results. These results indicate that the measured prebuckling strain responses, for the most part, fall on or within the predicted response bounds. However, the results also indicate that the predicted post-collapse strain response can be very sensitive to specimen parametric uncertainties. In addition, some discrepancy is expected based on sensitivity of the strain measurement in a region that exhibits large magnitude bending gradients. The measured and predicted prebuckling strain results for orthotropic shells $\mathrm{C} 1$ and $\mathrm{C} 2$ show similar agreement (not shown here).

\section{High-fidelity Models and Analysis-Based Buckling Knockdown Factors}

Modern nonlinear analysis tools, advanced measurement and test technologies, and improved understanding of the effects of imperfections on the buckling of thin-walled shells have enabled the development of high-fidelity structural models that can predict the buckling response of these shells to a high degree of accuracy. Validated high-fidelity models, such as those presented in 
section IV, are enabling the development of new analysis-based design knockdown factors for buckling-critical structures. Selected results that illustrate the use of high-fidelity models for the development of analysis-based knockdown factors are presented in this section. First, measured initial geometric imperfection data from six graphite-epoxy shells similar to shells C1-C3 are used to determine a manufacturing-process-specific imperfection signature for these shells. The imperfection signature represents a "first-approximation" imperfection shape that is suitable for developing preliminary-design data. This imperfection signature is then used as input into nonlinear finite-element analyses to predict the buckling response of a family of shells, based on shell C3, with different simulated imperfection shapes. These results presented in this section are documented in Reference 18.

\section{Manufacturing Imperfection Signature}

Shell-wall geometric imperfections that had been measured previously (see Refs. 15-17) for the six laminated-composite shells were used to derive a manufacturing-process-specific "first approximation" imperfection signature for this type of structure. First, a reference or "best-fit" cylinder for the initial imperfection measurement data for each shell was determined. Specifically, a data reduction program that uses the method of least squares was used to compute the rigid-body translations and rotations and the mean radius of each cylinder. Then, the measured radial imperfections, deviations from a perfect cylinder, were calculated with respect to the corresponding new best-fit cylinder. The measured imperfection data was then represented by a two-dimensional half-wave Fourier cosine series given by

$$
w_{o}(x, \theta)=t_{n o m} \sum_{k=0}^{N} \sum_{l=0}^{N} \cos \frac{k \pi x}{L}\left(A_{k l} \cos l \pi \theta+B_{k l} \sin l \pi \theta\right)
$$

where $R, L$, and $t_{n o m}$ are the shell radius, length and wall thickness; $\mathrm{x}$ and $\mathrm{y}$ are the axial and circumferential coordinates; $A_{k l}$ and $B_{k l}$ are amplitudes of the different modal components in the Fourier series; and $k$ and $l$ are integers denoting the number of axial half-waves and circumferential fullwaves, respectively. Using a standard representation of this type for the measured imperfection enables convenient analysis and comparison of different imperfection distributions from different shells and different manufacturing processes. For example, a plot of the coefficient distribution of a half-wave Fourier cosine representation of the measured geometric imperfection shape for shell specimen $\mathrm{C} 3$ is presented in Fig. 16. The results indicate that the largest-magnitude component of the imperfection is the $k=0$ and $l=2$ component and that the magnitudes of the other components decrease rapidly as the wave numbers $k$ and $l$ increase. The $k=0$ and $l=2$ component corresponds to a cylinder with an elliptical crosssection that has a magnitude of $1.2 t_{\text {nom }}$. The next largest component is associated with $k=1$ and $l$ $=2$ and has a magnitude of approximately $0.2 t_{\text {nom. }}$. In general, the dominant coefficients are associated with the long wave-length imperfections in the circumferential and axial directions. Coefficients for values of $k$ and $l>10$ have a small influence on the overall imperfection shape, however, the short wave-length components are retained in the imperfection model since these modes may be associated with high imperfection sensitivity for the shells considered herein even though their magnitudes are generally very small. Results for shells C1, C2, and C4-C6 indicate similar coefficient distributions and suggest that a manufacturing-process-specific imperfection signature for graphite-epoxy laminated-composite shells can be established in terms of a 
characteristic Fourier coefficient distribution and range. To this end, a mean imperfection shape and a corresponding standard deviation to the imperfection shape were determined based on the measured imperfection data from the six shells, $\mathrm{C} 1$ - C6. The mathematical expectation or mean value of $w_{o}(x, \theta)$ is given by

$$
\mu_{w}(x, \theta)=t_{n o m} \sum_{k=0}^{N} \sum_{l=0}^{N} \cos \frac{k \pi x}{L}\left(\left\langle A_{k l}\right\rangle \cos l \pi \theta+\left\langle B_{k l}\right\rangle \sin l \pi \theta\right)
$$

where $\langle\ldots\rangle$ denotes the mean value. A contour plot of the normalized mean geometric imperfections $\mu_{w}(x, \theta)$ is shown in Fig. 17. The mean shell-wall imperfection $\mu_{w}$ is normalized by the nominal shell-wall thickness $t_{\text {nom }}=0.04$ inches. These results indicate that the initial geometric shell-wall imperfection is periodic in the circumferential direction, exhibits slight variations in the axial direction, and is similar in character to the measured imperfection shape for shell C3 shown in Fig. 3. The corresponding standard deviation of the geometric imperfection shape $\sigma_{w}(x, \theta)$ is defined by

$$
\sigma_{w}^{2}\left(w_{o}(x, \theta)\right)=\left\langle\left(w_{o}(x, \theta)-\left\langle w_{o}(x, \theta)\right\rangle\right)^{2}\right\rangle
$$

A contour plot of the corresponding normalized standard deviation of the geometric imperfection shape is shown in Fig. 18. The results indicate that the maximum standard deviation is equal to $2.172 t_{n o m}$ and is primarily associated with long wave-length components of the imperfection shape.

\section{Response of Compression-Loaded Shells}

The effects of four different imperfection shapes on the response of shell C3 are presented. Specifically, the effects of actual measured shell-wall imperfections, and three simulated imperfections that are based on the imperfection signature. The simulated imperfections include the mean imperfection and the mean imperfection plus or minus one standard deviation. A shell with the mean imperfection shape is denoted by $\mu_{w}$; a shell with the mean imperfection shape plus one standard deviation is denoted by $\mu_{w}+\sigma_{w}$; and a shell with the mean imperfection shape minus one standard deviation is denoted by $\mu_{w}-\sigma_{w}$. Load-shortening response curves and shell deformations are presented subsequently for the quasi-isotropic shell C3 that illustrate the overall response characteristics for the compression-loaded shell with the four different imperfection shapes.

Four predicted load-shortening response curves are presented for shell C3 in Fig. 19 and include results for a shell with the actual measured imperfection shape;. The axial load $P$ and endshortening $\Delta$ are normalized with respect to the linear bifurcation buckling load of the geometrically perfect nominal shell, $P_{\text {bif }}=42,590 \mathrm{lbf}$, and the nominal shell-wall thickness, $t_{\text {nom }}$ $=0.04$ in, respectively. The load-end-shortening curves indicate a linear prebuckling response.

The general instability occurs in the shell at normalized axial load levels that range from $P / P_{b i f}$ $=0.855$, for a shell with $\mu_{w}+\sigma_{w}$ imperfection shape, to 0.943 for the shell with a $\mu_{w}-\sigma_{w}$ imperfection shape.

Initial buckling deformations for shell $\mathrm{C} 3$ with the mean imperfection shape, the $\mu_{w}+\sigma_{w}$ 
imperfection shape, and $\mu_{w}-\sigma_{w}$ are presented in Figs. 20a through 20c and indicate similar response characteristics to those exhibited by the shell with the actual measured imperfection. In particular, the initial buckling deformation response of a shell with the mean imperfection and the $\mu_{w}+\sigma_{w}$ imperfection are similar to that exhibited by the shell with the actual measured imperfection and are characterized by a localization of a single buckle near one end of the shell. These results are significant because they suggest that the use of a mean imperfection shape and the corresponding standard deviation can bound the experimental buckling loads as well as predict physically meaningful response characteristics such as the buckling deformations. The correlation would improve still further if other measured imperfections were included in the finite-element model; e.g., measured thickness variation, material property variation, and loading nonuniformities, as shown in Refs. 17 and 18. Specifically, the measured thickness imperfection has been shown to account for an additional 5-10\% reduction in the buckling load. In comparison, the lower-bound design recommendation given in NASA SP-8007 for the corresponding isotropic shell with $R / t=200$ is equal to $P_{c r} / P_{b i f}=0.47$, which is very conservative, as expected.

\section{Concluding Remarks}

The modern high-fidelity analysis approach offers a relatively affordable method to develop updated design knockdown factors for modern buckling-critical shell structures. The suggested design and high-fidelity analysis procedures presented herein should be used with a selected number of carefully conducted experiments that would be used to validate the design and analysis results. This approach can help avoid testing the large number of replicates of a design needed to develop empirical design factors. Furthermore, this nonlinear analysis procedure can be used as a parametric tool in the early stages of a design development program to determine the sensitivity of the response characteristics of a specific design to a number of different types of imperfections or differences in the idealized as-designed shell structure and the actual asmanufactured shell structure. Since this nonlinear analysis procedure can predict the local stresses and strains in a shell for any point in the load-response history of the shell, failure of the shell due to local stress or strain gradients could also be predicted.

In recent years, a comprehensive project has been established at NASA to develop and validate new analysis-based shell buckling knockdown factors and design recommendations for modern launch vehicle structures. These new knockdown factors are based on the results from highfidelity analysis tools such as the ones presented here and are being rigorously validated through sub-scale and full-scale structural tests. These new factors will account for the effects of geometric imperfections, loading nonuniformities, orthotropy, longitudinal welds, and combined mechanical, thermal and pressure loads typically found in large metallic cylindrical structures. It is expected that the new factors will enable significant mass reduction in the next generation of NASA launch vehicles as well as reduce development cost and risk. The STAGS finite-element code has played a prominent role in all phases of the development of high-fidelity analysis methods for buckling-critical structures and has enabled the development of these new analysisbased knockdown factors. It is with this in mind that one can recognize the continued need for research-oriented codes such as STAGS as a development test bed and benchmark for future 
high-fidelity physics-based models that will be needed to meet the next generation of structural analysis challenges.

\section{Acknowledgments}

In memory of Dr. Charles C. Rankin. A long-time developer of the STAGS finite-element code and good friend.

This work was conducted as part of the NASA Engineering and Safety Center (NESC) Shell Buckling Knockdown Factor Project, NESC Assessment \#:07-010-E.

\section{References}

1. von Kármán, T. and Tsien, H. S., "The Buckling of Thin Cylindrical Shells Under Axial Compression," Journal of the Aeronautical Science, Vol. 8, No. 8, June 1941, pp. 303-312.

2. Donnell, L. H. and Wan, C. C., "Effect of Imperfections on Buckling of Thin Cylinders and Columns Under Axial Compression.," Journal of Applied Mechanics, Vol. 17, No. 1, March 1950, pp. 73-83.

3. Koiter, W. T., On the Stability of Elastic Equilibrium. (in Dutch), H. J. Paris, Amsterdam, Holland, 1945; translation available as AFFDL-TR-70-25, February, 1970, Wright-Patterson Air Force Base.

4. "Buckling of Thin-Walled Circular Cylinders," NASA Space Vehicle Design Criteria, NASA SP-8007, September 1965 (Revised 1968).

5. "Buckling of Thin-Walled Truncated Cones," NASA Space Vehicle Design Criteria, NASA SP-8019, September 1968.

6. "Buckling of Thin-Walled Doubly Curved Shells," NASA Space Vehicle Design Criteria, NASA SP-8032, August 1969.

7. Peterson, James P.: Buckling of Stiffened Cylinders in Axial Compression and Bending - A Review of Test Data. NASA TN D-5561. 1969.

8 "Isogrid Design Handbook," NASA CR-124075, February 1973.

9. Budiansky, B, "Theory of Buckling and Postbuckling Behavior of Elastic Structures," Advances in Applied Mechanics , Vol. 14, Academic Press, 1974, pp. 1-65.

10. Árbocz, J., Babcock, C. D., Jr., "The Effects of General Imperfections on the Buckling of Cylindrical Shells," Journal of Applied Mechanics , Vol. 36, No. 1, pp. 28-38, 1969.

11. Árbocz, J. and Williams, J. G., "Imperfection Surveys on a 10-ft-Diameter Shell Structure," AIAA Journal , Vol. 15, 1977, pp. 949-952.

12. Árbocz, J., Babcock, C. D., Jr., "Computerized Stability Analysis Using Measured geometric Imperfections," Proceedings of the 12th Congress of the International Council of the Aeronautical Sciences , Munich, Germany, ICAS-80-20.0, pp. 688-701, 1980.

13. Árbocz, J., "The Imperfection Data Bank, a Means to Obtain Realistic Buckling Loads," 16 American Institute of Aeronautics and Astronautics 
Buckling of Shells- A State-of-the-Art Colloquium , E. Ramm, ed., Springer Verlag, Berlin, 1982, pp. 535-567.

14. "NASTRAN User's Manual," NASA-SP-222(08),Vol. 2, June, 1986.

15. Rankin, C. C., Brogan, F. A., Loden, W. A., and Cabiness, H. D., "STAGS Users Manual, Version 4.0," Lockheed Martin Missiles \& Space Co., Inc., Advanced Technology Center, Report LMSC P032594, 1999.

16. Starnes, J. H., Jr., Hilburger, M. W., and Nemeth, M. P., "The Effects of Initial Imperfections on the Buckling of Composite Cylindrical Shells," Composite Structures - Theory and Practice , P. Grant and C. Q. Rousseau, eds., ASTM STP 1383, 2000, pp. 529- 550.

17. Hilburger, M. H., and Starnes, J. H., Jr., "High-Fidelity Analysis of Compression-loaded Composite Shells," Proceedings of the 42st AIAA/ASME/ASCE/AHS/ASC Structures, Structural Dynamics, and Materials Conference, Seattle, WA. AIAA Paper No. 2001-1394, 2001.

18. Hilburger, M.W.; Starnes, J.H., Jr.: Effects of Imperfections on the Buckling Response of Compression-Loaded Composite Shells, International Journal of Non-Linear Mechanics, vol. 37, no. 4-5, pp. 623-643, 2002.

19. Nemeth, M. P., Britt, V. O., Young, R. D., Collins, T. J., and Starnes, J. H., Jr., "Nonlinear Behavior of Space Shuttle Super-light-weight Liquid-Oxygen Tank Under Prelaunch Loads," Journal of Spacecraft and Rockets, Vol. 36, No.6, November-December, 1999, pp. 788-803.

20. Nemeth, M. P., Young, R. D., Collins, T. J., and Starnes, J. H., Jr., "Nonlinear Behavior of Space Shuttle Super-light-weight Liquid-Oxygen Tank Under End-of-Flight Loads," Journal of Spacecraft and Rockets, Vol. 36, No.6, November-December, 1999, pp. 828-835.

21. Young, R. D., Nemeth, M. P., Collins, T. J., and Starnes, J. H., Jr.,"Nonlinear Behavior of Space Shuttle Super-light-weight Liquid-Oxygen Tank Under Booster Ascent Loads," Journal of Spacecraft and Rockets, Vol. 36, No.6, November-December, 1999, pp. 820-827.

22. Nemeth, M. P., Young, R. D., Collins, T. J., and Starnes, J. H., Jr., "Effects of WeldingInduced Imperfections on Behavior of Space Shuttle Super-light-weight Liquid-Oxygen Tank," Journal of Spacecraft and Rockets, Vol. 36, No.6, November-December, 1999, pp. 812-819.

23. Nemeth, M. P., Young, R. D., Collins, T. J., and Starnes, J. H., Jr., "Effects of Initial Geometric Imperfections on the Nonlinear Response of the Space Shuttle Super-light-weight Liquid-Oxygen Tank," International Journal of Non-Linear Mechanics, Vol. 37, No.4-5, June, 2002, pp. 723-744.

24. Hilburger, M.W.; Nemeth, M.P.; Starnes, J.H.,Jr., "Shell Buckling Design Criteria Based on Manufacturing Imperfection Signatures,” AIAA Journal, vol. 44, no. 3, March 2006. 
25. Hilburger, M. W., "Developing the Next Generation Shell Buckling Design Factors and Technologies," Proceedings of the $53^{\text {rd }}$ AIAA/ASME/ASCE/AHS/ASC Structures, Structural Dynamics, and Materials Conference, Honolulu, HI, AIAA Paper N0. 2012-1686, 2012.

26. Hilburger, M.W., "Numerical and Experimental Study of the Compression Response of Composite Cylindrical Shells with Cutouts," Ph.D. dissertation, Department of Aerospace Engineering, University of Michigan, Ann Arbor, MI, Feb. 1998.

27. Weiland, T. M., Morton, J., and Starnes, J.H., "Scaling Effects in Buckling, Postbuckling and Crippling of Graphite-Epoxy Z-Section Stiffeners," Virginia Polytechnic Institute and State University, Center for Composite Materials and Structures, Report CCMS-92- 25, September 1992.

28. Riks, E., "Progress in Collapse Analysis," Journal of Pressure Vessel Technology, Vol. 109, 1987, pp. 27-41.

29. Park, K. C., "An Improved Stiffly Stable Method for Direct Integration of Nonlinear Structural Dynamics," Journal of Applied Mechanics, Vol. 42, June 1975, pp. 464-470.

30. Riks, E., Rankin, C. C., and Brogan, F. A., "On the Solution of Mode Jumping Phenomena in Thin-Walled Shell Structures," Computer Methods in Applied Mechanics and Engineering, Vol. 136 (1-2), 1996, pp. 59-92. 


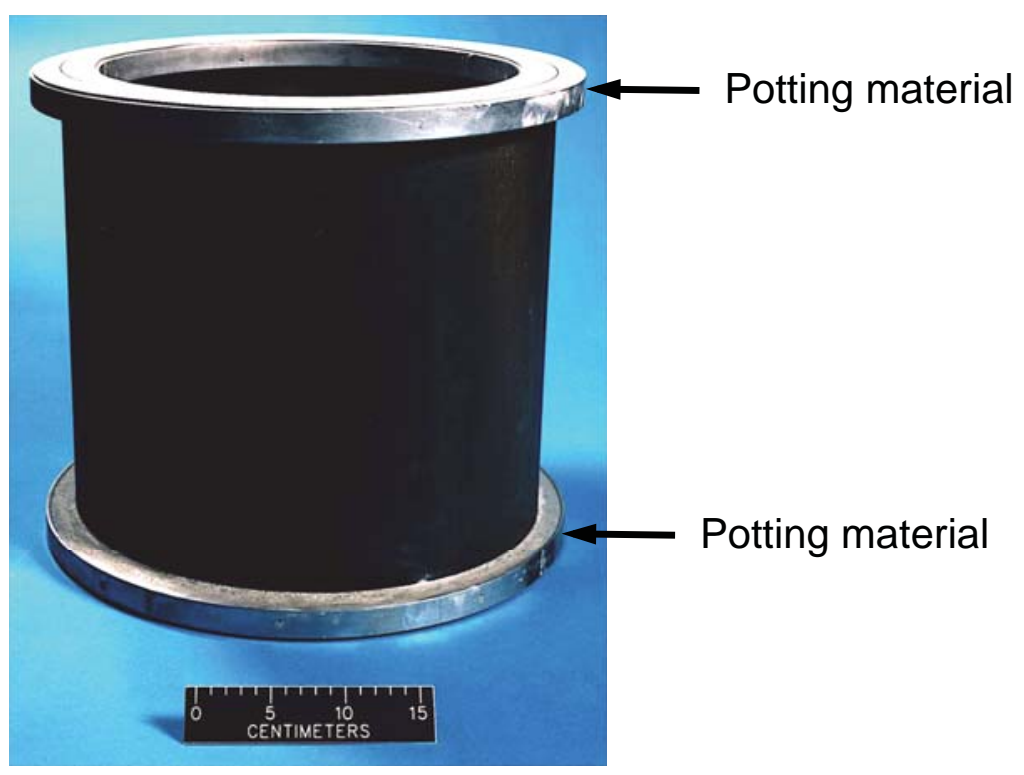

Figure 1. Typical laminated composite cylinder.
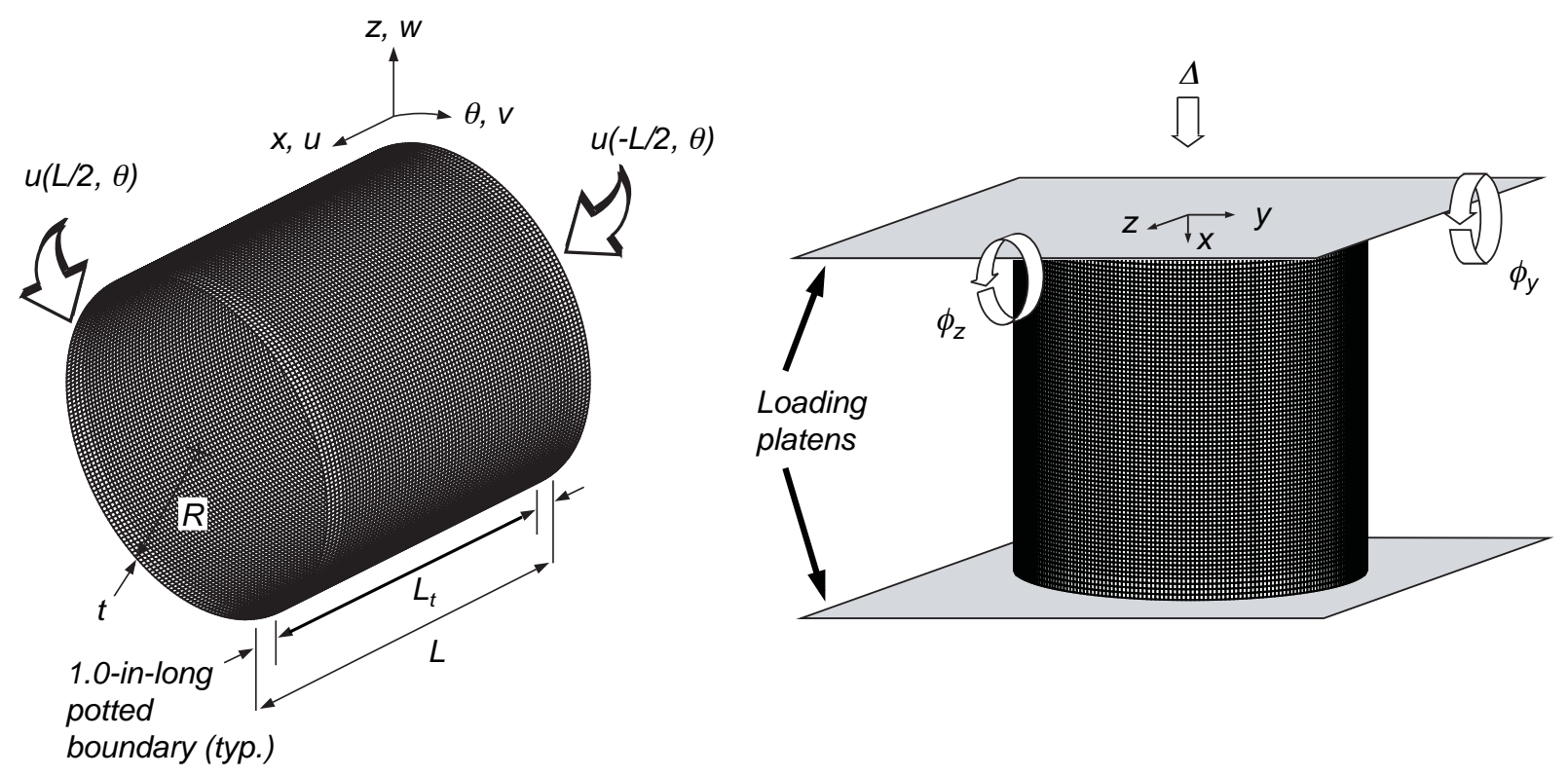

Figure 2. Finite-element model. 


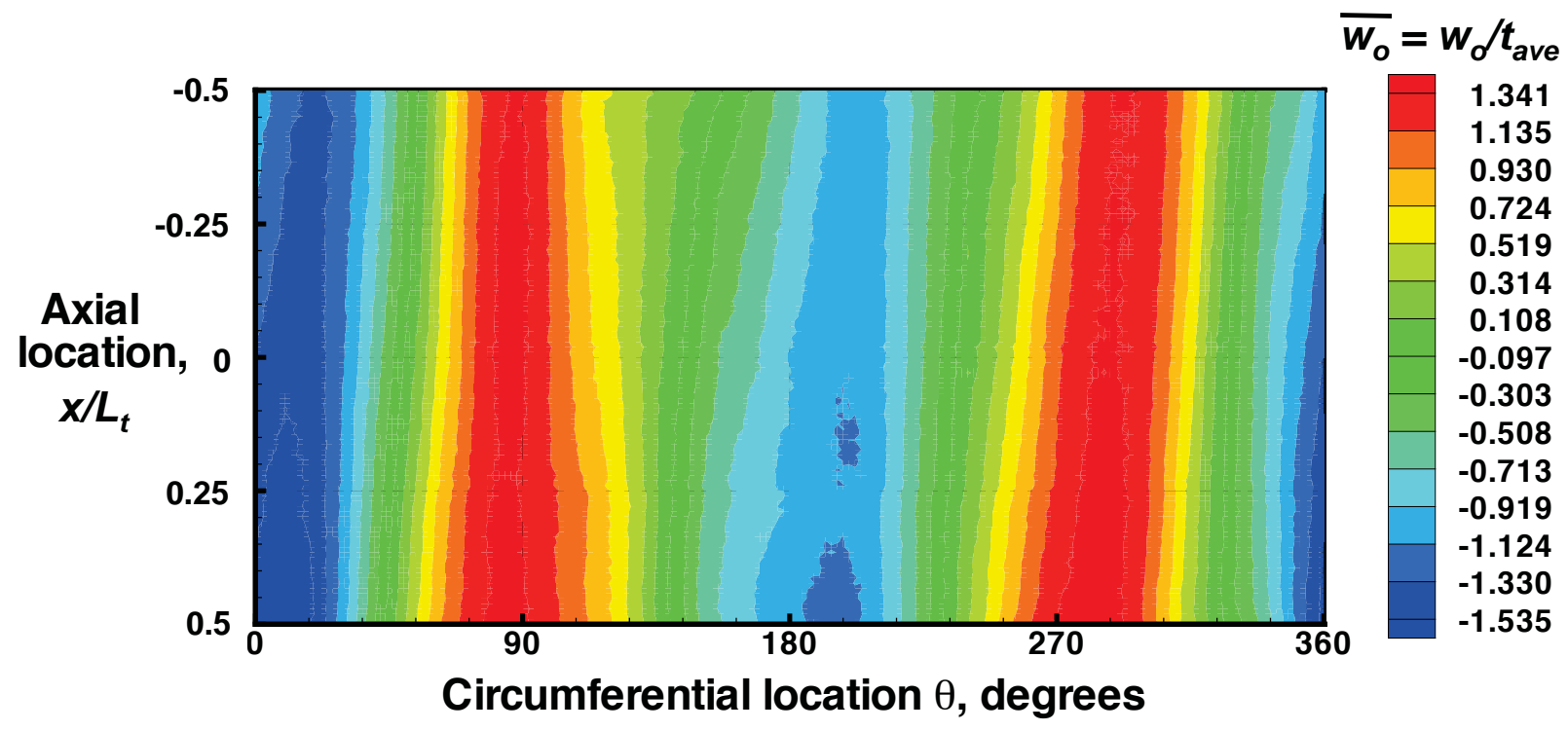

Figure 3. Measured geometric imperfection of quasi-isotropic shell C3.

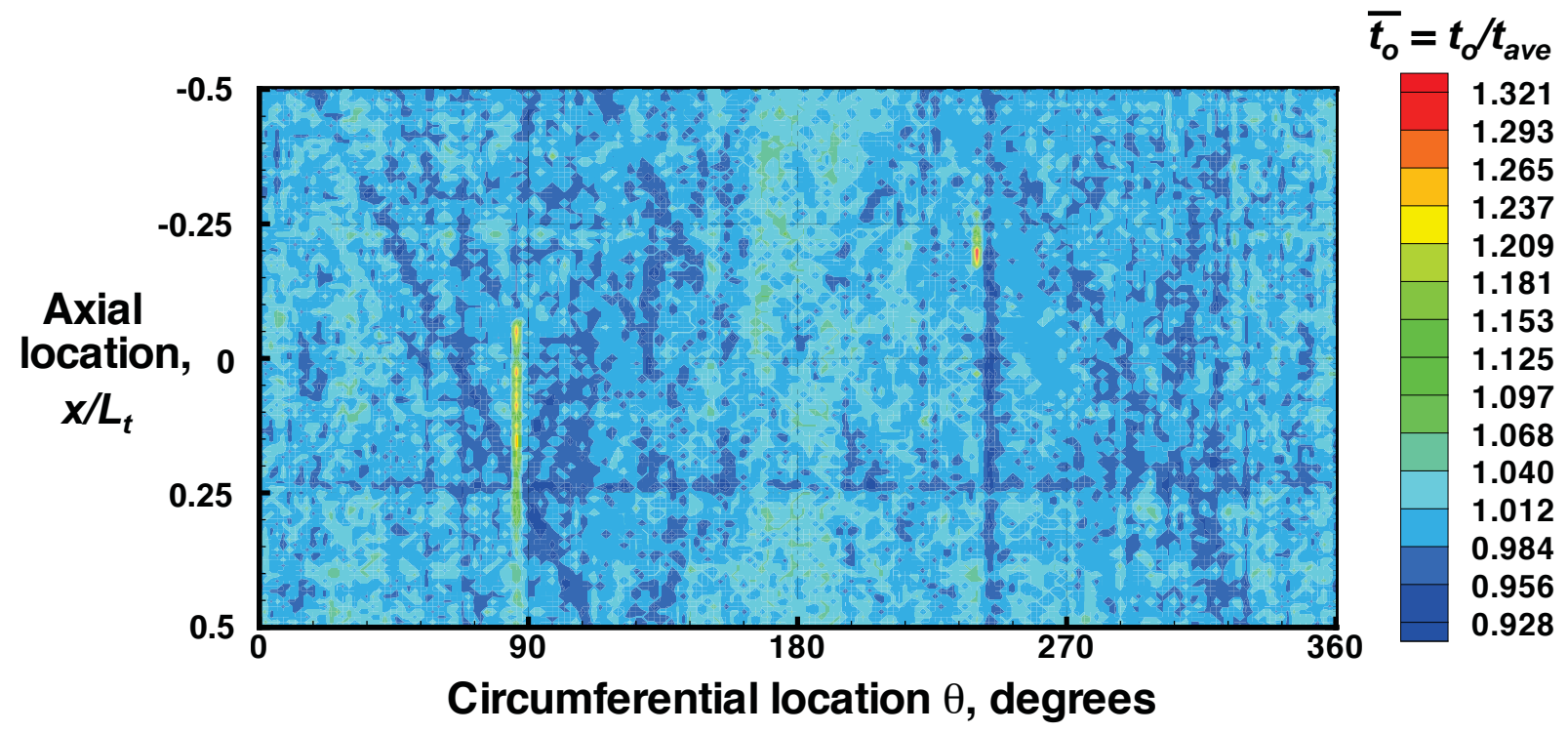

Figure 4. Measured shell-wall thickness of quasi-isotropic shell C3. 


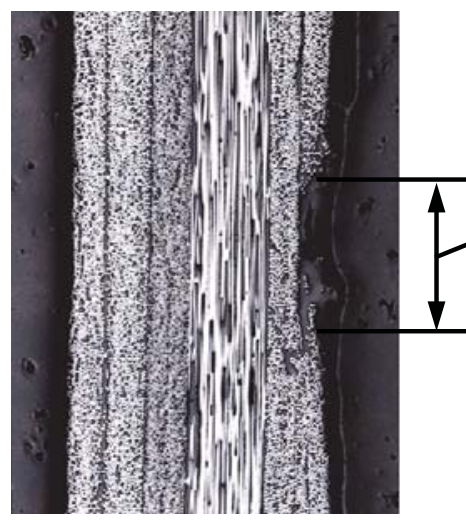

a) Ply-gap in a $45^{\circ}$ outersurface lamina ply

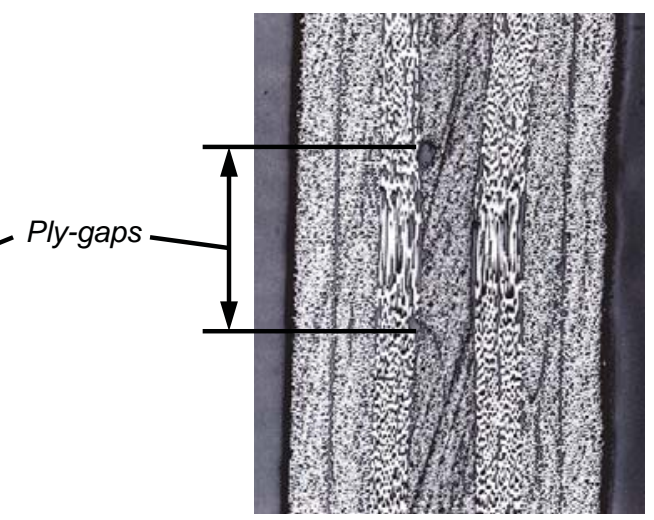

b) Ply-gap in a $90^{\circ}$ interior lamina ply

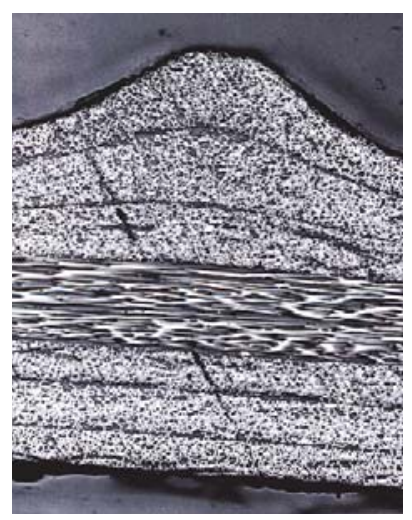

c) Outer-surface ridge

Figure 5. Typical lamina ply-gap and outer-surface ridges in 8-ply laminated composite cylinders.
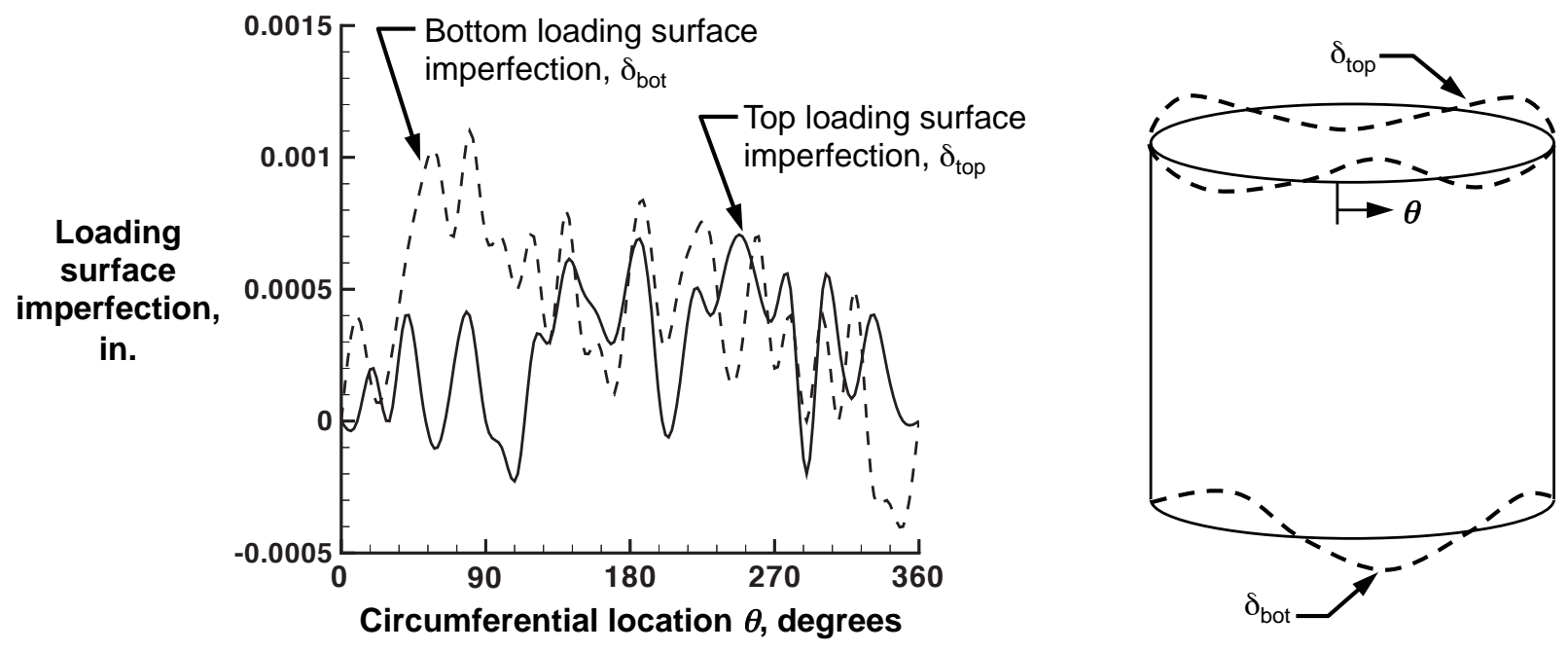

Figure 6. Measured loading surface imperfection for shell C3. 

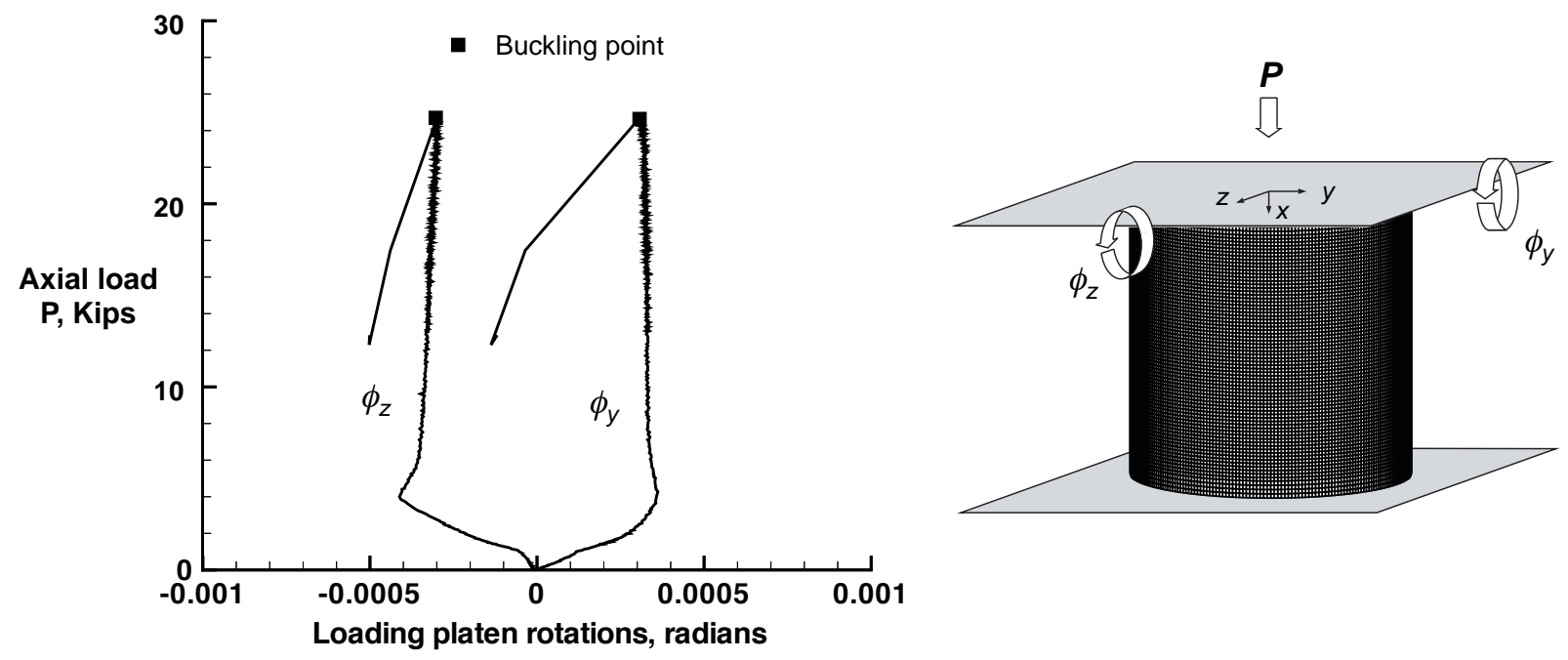

Figure 7. Typical measured loading platen rotations during experimental testing.

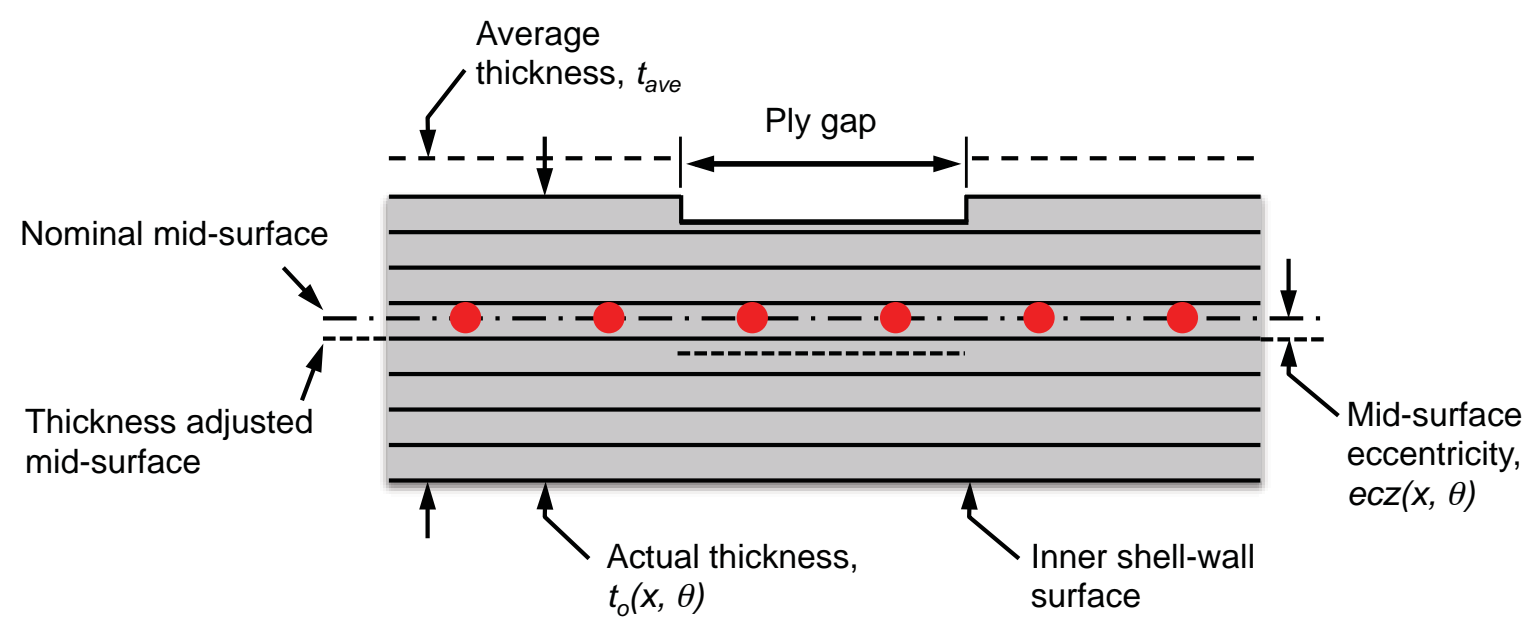

Finite element integration point

Figure 8. Idealized ply-gap model detail. 

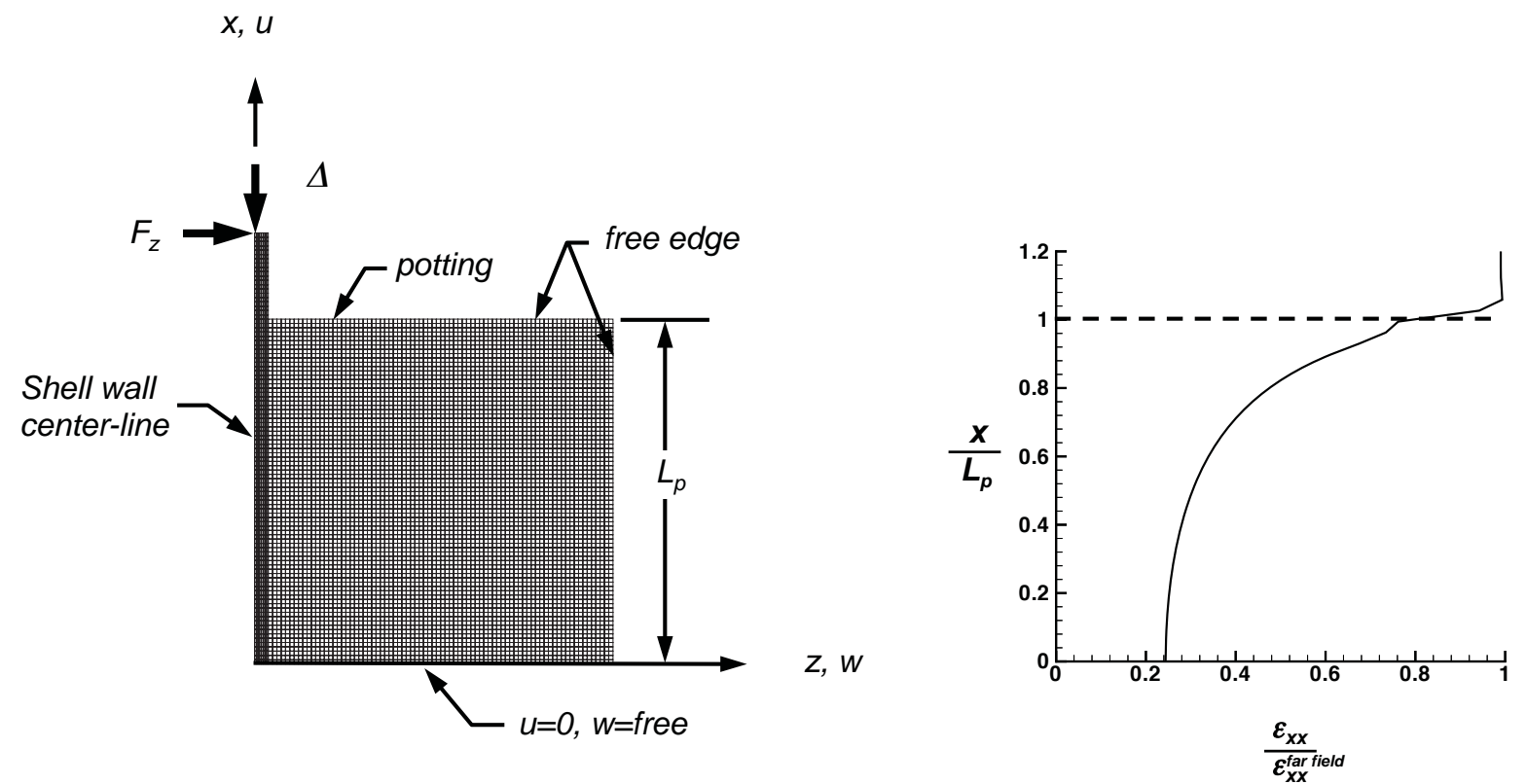

Figure 9. Finite-element model of the potting-shell detail and typical predicted axial strain distribution at the mid-surface of the shell wall.

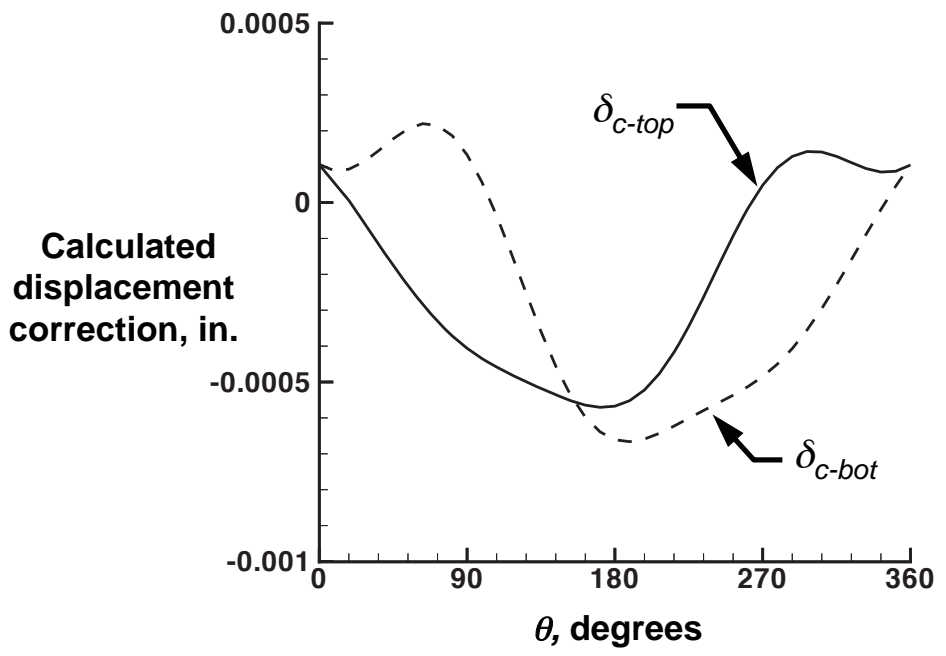

Figure 10. Numerically predicted displacement corrections $\delta_{c-t o p}$ and $\delta_{c-b o t}$ for the top and bottom loading surface, respectively, inferred from experimentally measured axial strains for shell C3. 


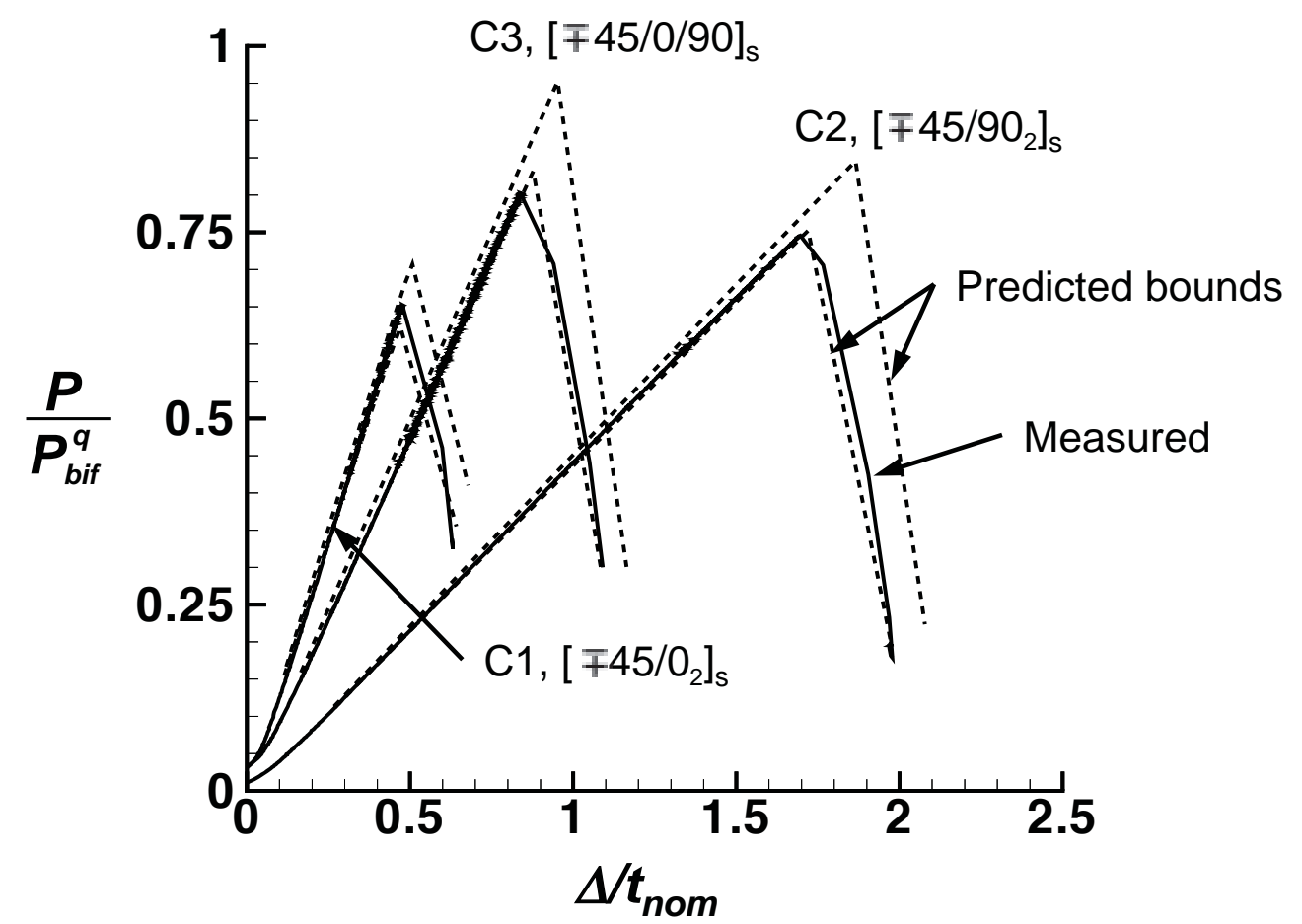

Figure 11. Predicted and measured load-end-shortening response curves for shells C1, C2, and C3. Predicted results represent response bounds.

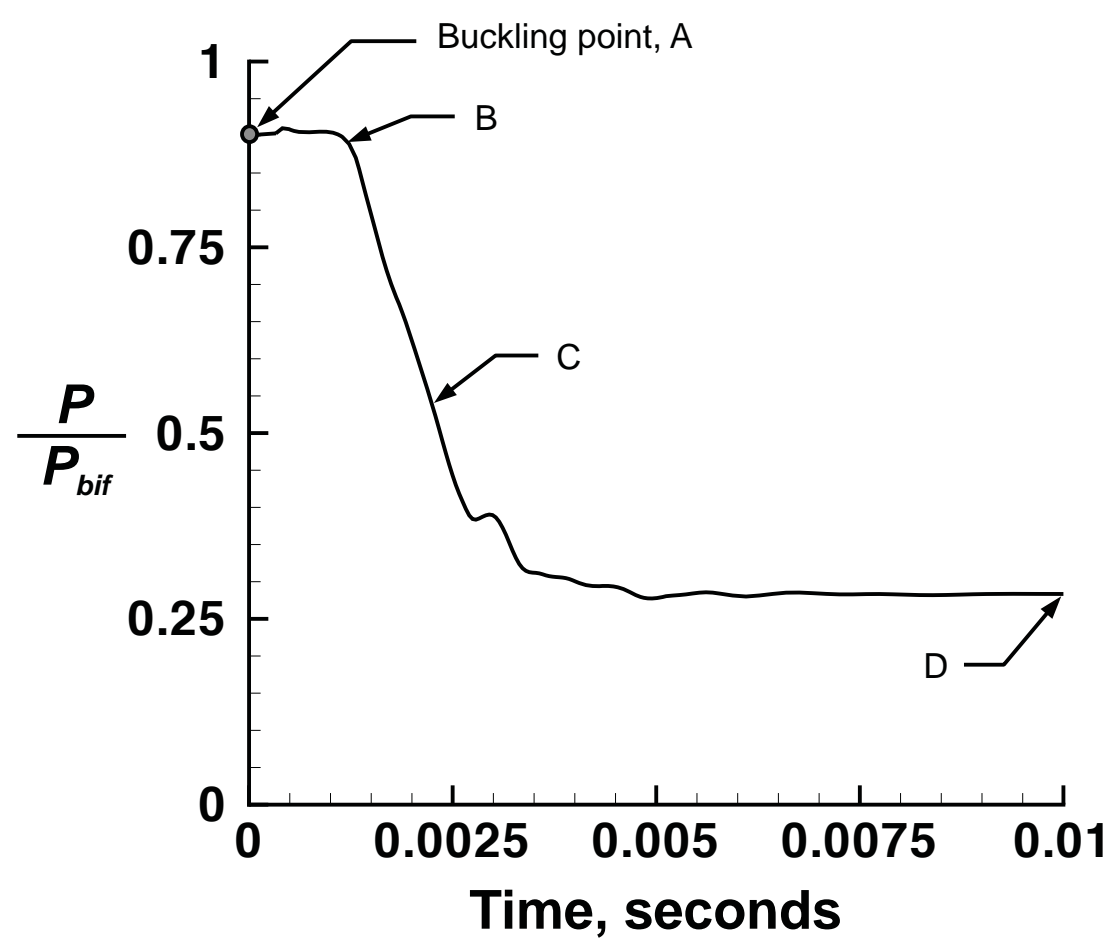

Figure 12. Predicted load versus time history for the transient collapse of geometrically imperfect shell C3. 


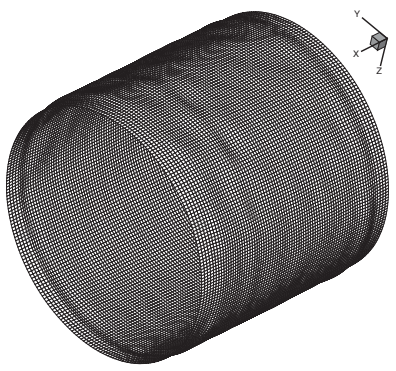

a) Prebuckling deformations b) Initial buckling Time $=0.0$ seconds

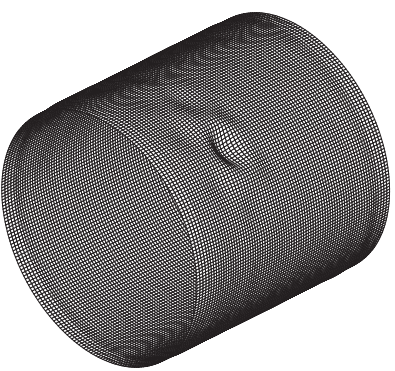

deformations

Time $=0.00143$ seconds

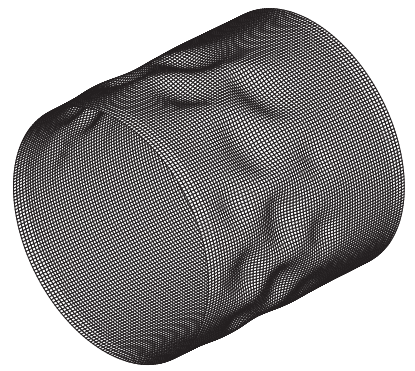

c) Transient buckling deformations

Time $=0.00238$ seconds

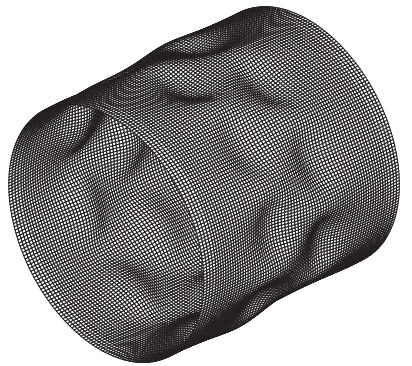

d) Post-buckling deformations Time $=0.01$ seconds

Figure 13. Predicted buckling response for an imperfect compression-loaded quasi-isotropic shell C3.

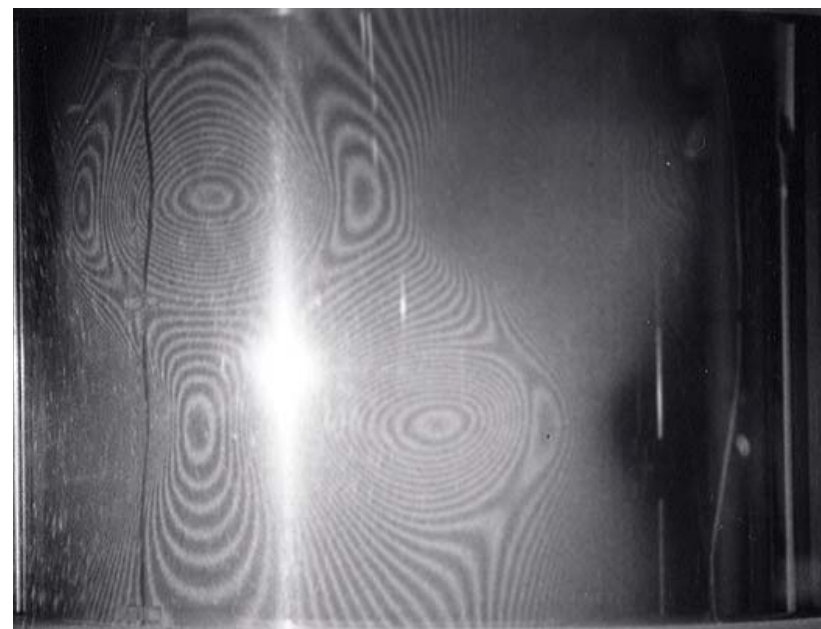

a) Post-buckling moiré fringe pattern

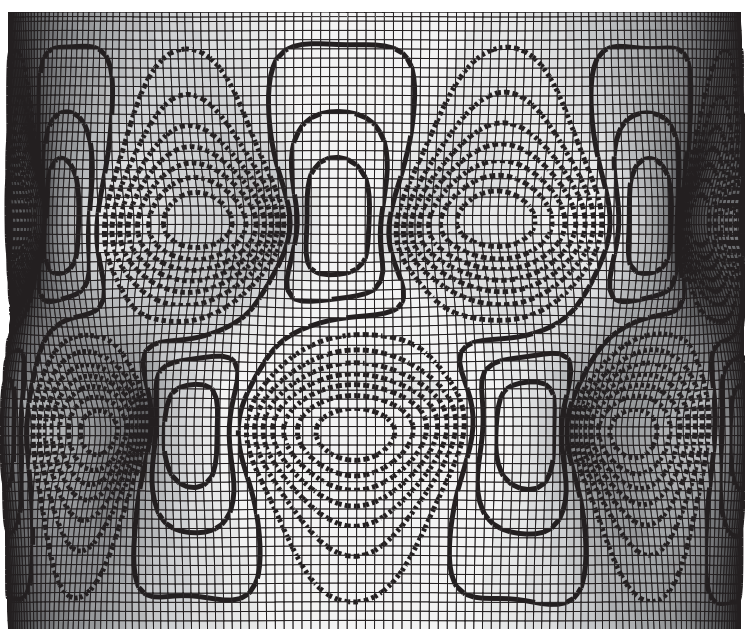

b) Predicted post-buckling radial displacements

Figure 14. Observed and predicted initial post-buckling radial displacements for shell $\mathrm{C} 2$ (similar to the response for shell C3). 


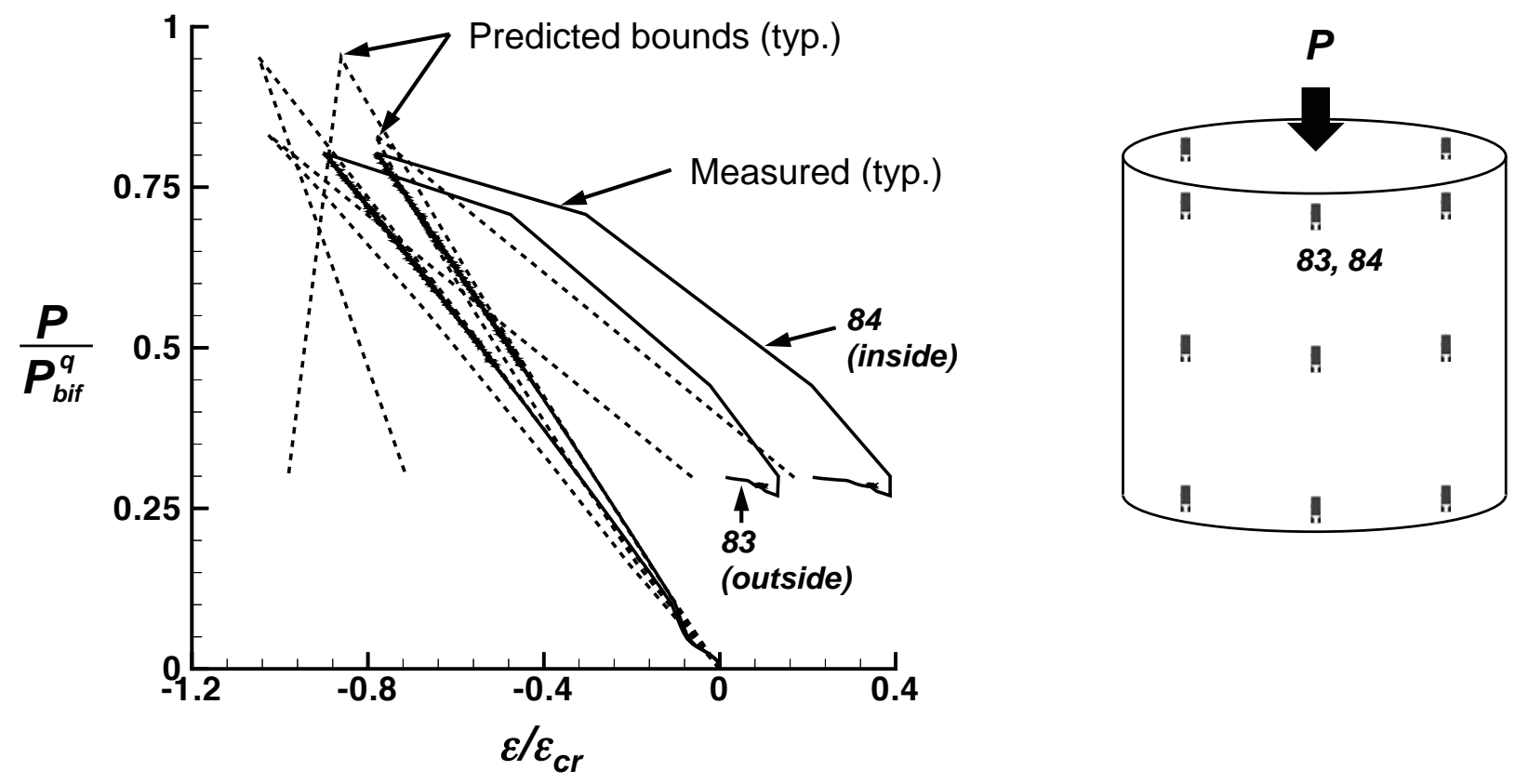

Figure 15. Typical predicted and measured back-to-back strain response for quasi-isotropic shell C3.
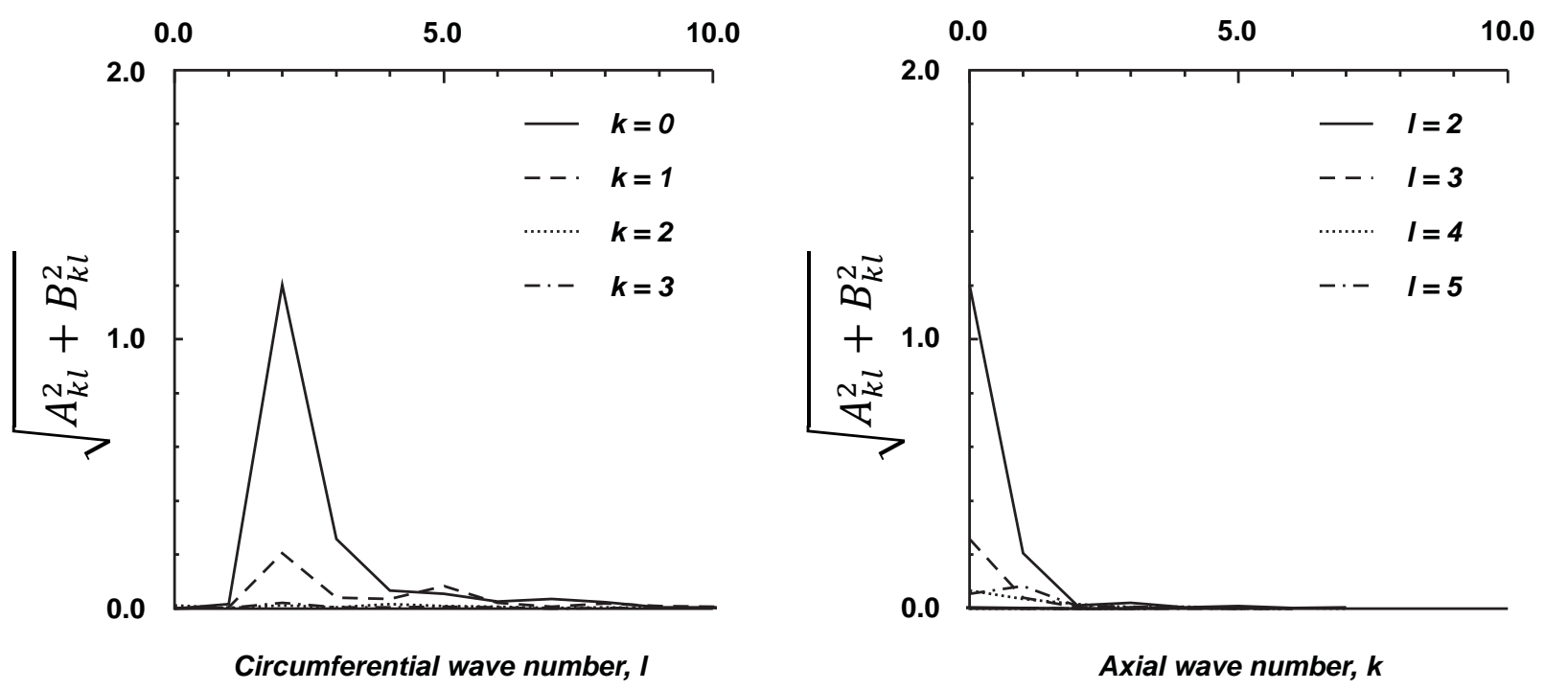

Figure 16. Coefficient distribution of a Fourier series representation of the measured geometric imperfection for shell C3. 


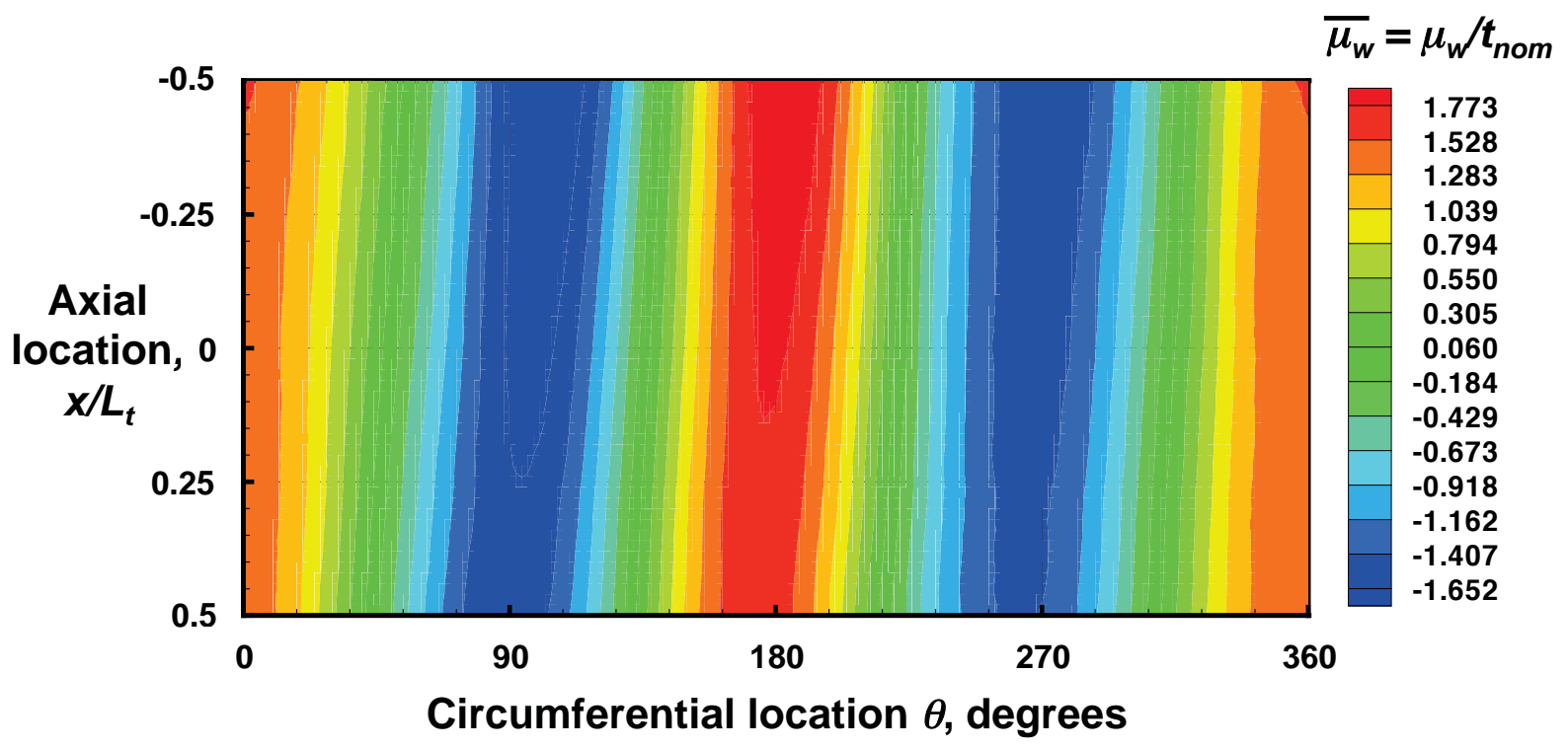

Figure 17. "First approximation" imperfection signature.

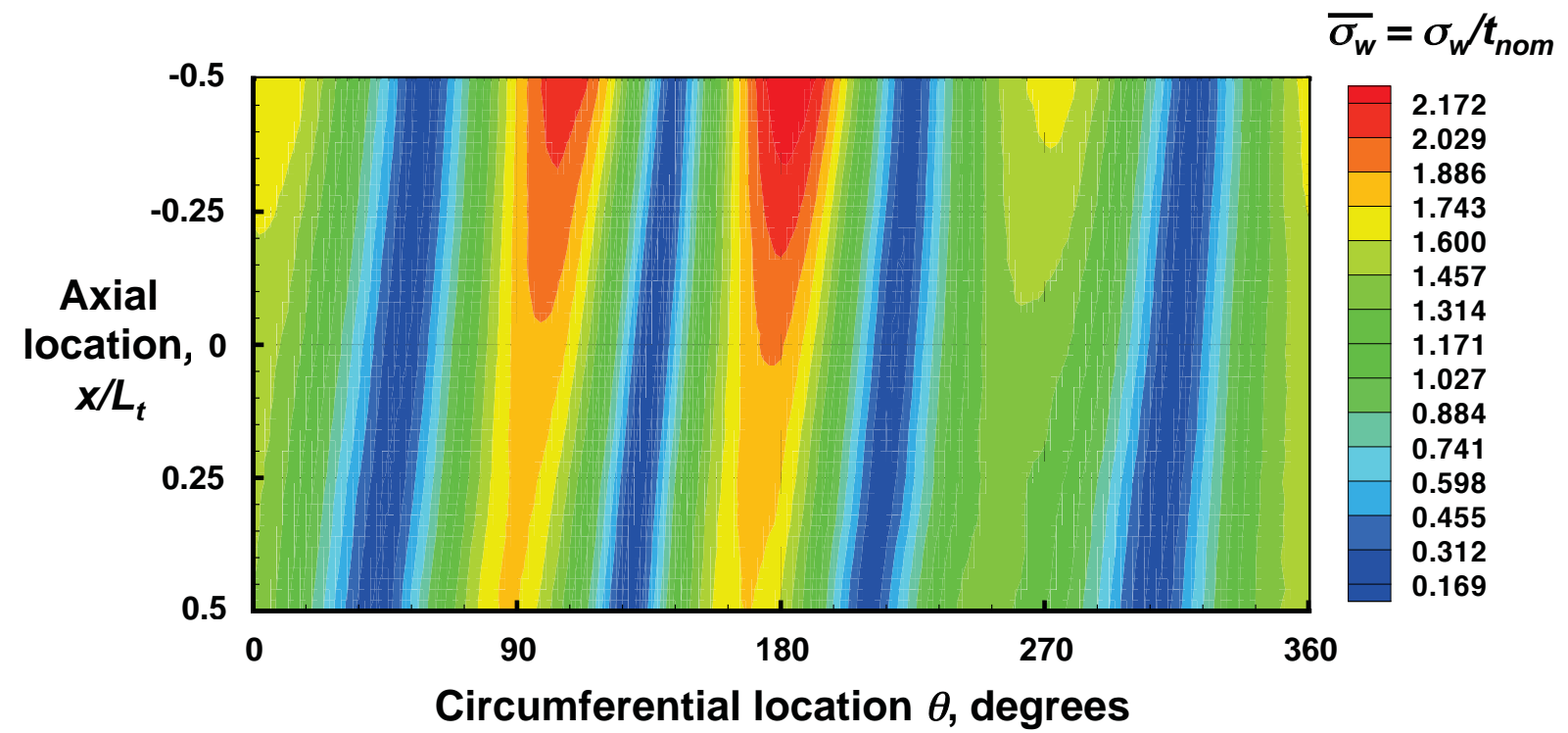

Figure 18. Standard deviation to the imperfection signature shown in Figure 17. 


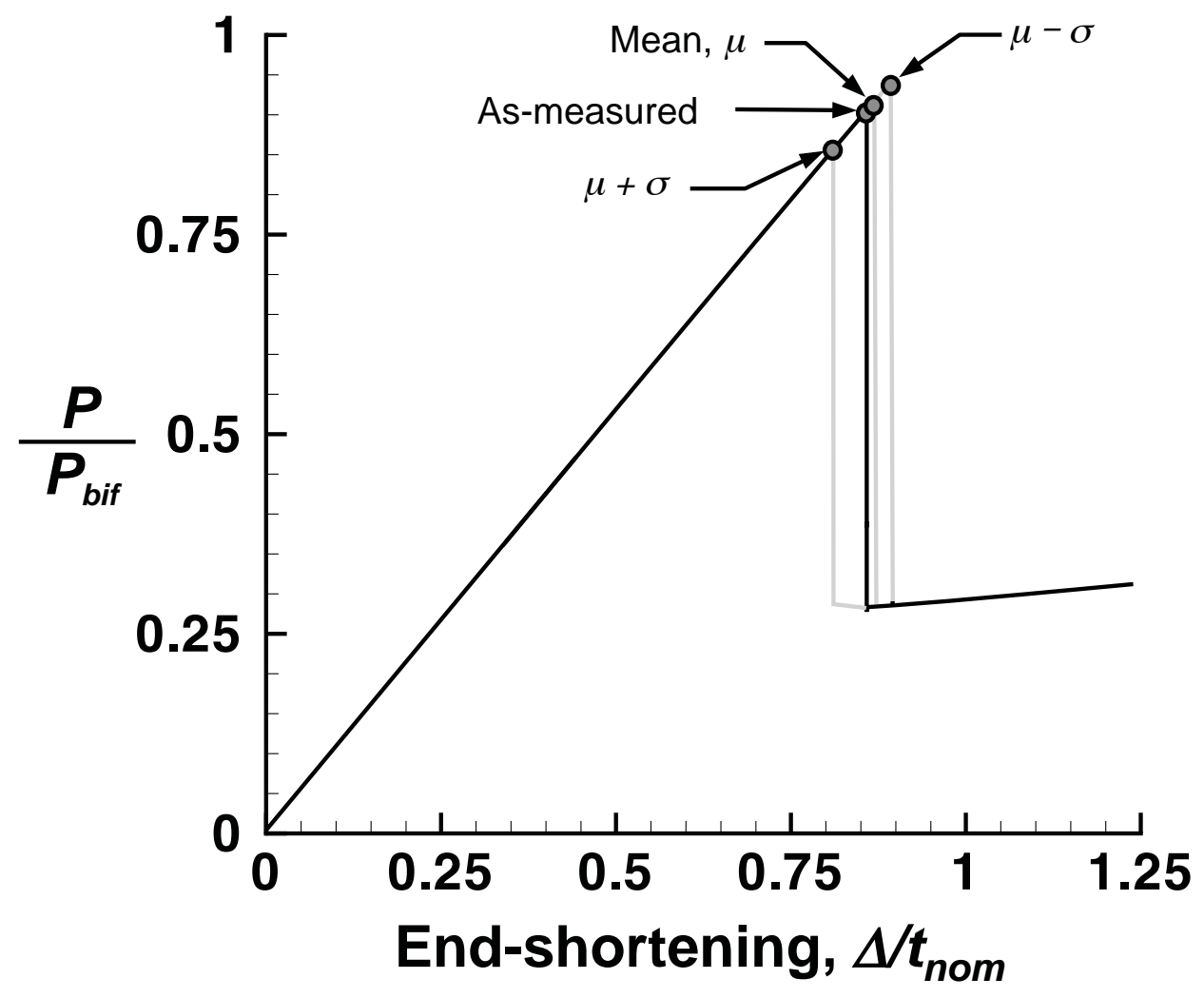

Figure 19. Predicted nonlinear response for the compression-loaded shell C3 with selected initial imperfection shapes.

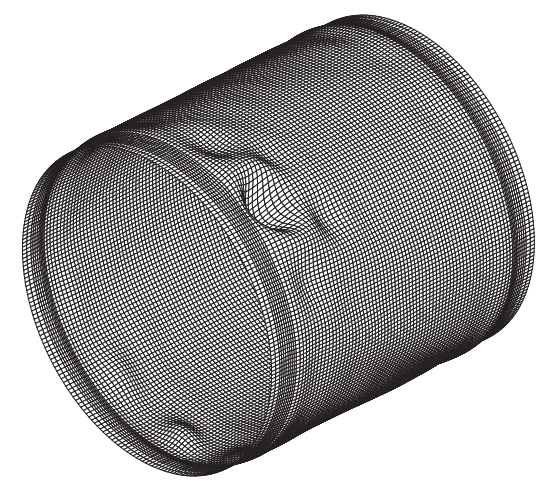

a) Mean imperfection, $\mu_{w}$ Time $=0.0011$ seconds $P / P_{b i f}=0.918$

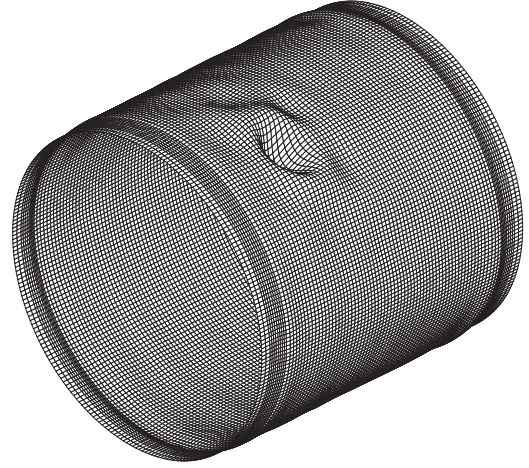

b) $\mu_{w}+\sigma_{w}$ imperfection Time $=0.0012$ seconds $P / P_{b i f}=0.855$

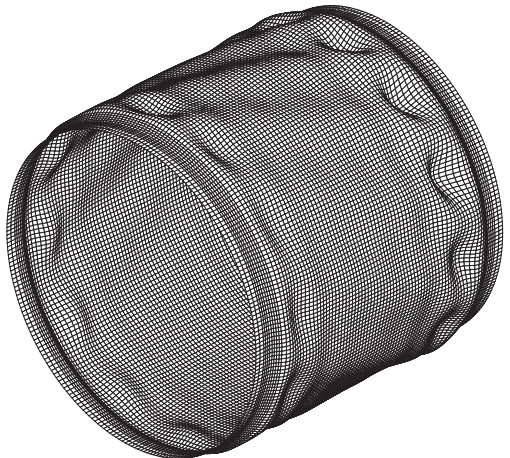

c) $\mu_{w}-\sigma_{w}$ imperfection Time $=0.0010$ seconds $P / P_{b i f}=0.943$

Figure 20. Predicted initial buckling deformation responses for the compression-loaded shell C3 with selected simulated initial imperfection shapes. 